Proyecciones Journal of Mathematics

Vol. 37, No 3, pp. 439-477, September 2018.

Universidad Católica del Norte

Antofagasta - Chile

\title{
A computer verification for the value of the Topological Entropy for some special subshifts in the Lexicographical Scenario
}

\author{
Solange Aranzubia \\ Universidad Central de Chile, Chile \\ Rubén Carvajal \\ Universidad de Santiago de Chile, Chile \\ and \\ Rafael Labarca \\ Universidad de Santiago de Chile, Chile \\ Received: July 201\%. Accepted : August 2018 \\ Abstract
}

The Lorenz Attractor has been a source for many mathematical studies. Most of them deal with lower dimensional representations of its first return map. An one dimensional scenario can be modeled by the standard two parameter family of contracting Lorenz maps. The dynamics, in this case, can be modeled by a subshift in the Lexicographical model. These subshifts are the maximal invariant set for the shift map in some interval. For some of them the extremes, of the interval, are a minimal periodic sequence and a maximal periodic sequence which is an iteration of the lower extreme (by the shift map). For some of these subshifts the topological entropy is zero. In this case the dynamics (of the respective Lorenz map) is simple. Associated to any of these subshifts (let call it $\Lambda$ ) we consider an extension (let call it $\Gamma$ ) of it that contains $\Lambda$ which also can be constructed by using an interval whose extremes can be defined by the extremes of $\Lambda$. For these extensions, we present here a computer verification of a result that compute its topological entropy. As a consequence, we can affirm: the longer the period of the periodic sequence is, then the lower complexity in the dynamics of the extension the associated map has.

2000 AMS Classification : Primary 37B10, 37B40, Secondary $37 E 05$.

Key words and phrases : Lexicographical world, topological entropy, maximal and minimal sequences, shift map, Lorenz Maps. 


\section{Introduction}

The topological theory of Dynamical Systems is a mathematical theory that started in the first quarter of the 20th century due, mainly, to the pioneer work by Poincaré which introduced the definition of the rotation number associated to an homeomorphism of the circle.

The main task of this topological theory is to study the dynamical behavior of a continuous map $f: X \rightarrow X$, where $X$ is a compact topological space. In the 1960's and in a remarkable article by R. L. Adler, A. G. Konheim and M. H. McAndrew (see [1]) these authors introduced the topological entropy of a map $f: X \rightarrow X$.

Let us assume that $(X, d)$ is a compact metric space. Let $C^{0}(X, X)$ be the set of continuous maps $f: X \rightarrow X$ endowed with the topology given by the metric $d_{0}(f, g)=\operatorname{Sup}\{d(f(x), g(x)) ; x \in X\}$. The topological entropy is a map $h_{\text {top }}: C^{0}(X, X) \rightarrow[0, \infty[$ that is not continuous (see [30]). The number $h_{t o p}(f)$ indicates the complexity of the dynamic of the map $f: X \rightarrow X$. In fact, the number $e^{h_{t o p}(f)}=\lambda_{f}$ has the following property: Given a minimal open covering of the space X, say $\mathcal{A}=\left\{A_{1}, A_{2}, \ldots A_{p}\right\}$. Let $\mathcal{A}_{n}=\left\{A_{i_{0}} \cap f^{-1}\left(A_{i_{1}}\right) \cap \ldots \cap f^{-(n-1)}\left(A_{i_{n-1}}\right) ; A_{i} \in \mathcal{A}\right\}$ be the open covering $\mathcal{A} \vee f^{-1}(\mathcal{A}) \vee \ldots \vee f^{-(n-1)}(\mathcal{A})$. Let $N\left(\mathcal{A}_{n}, f\right)$ be the small cardinality of an open subcover of $\mathcal{A}_{n}$ then $N\left(\mathcal{A}_{n}, f\right) \sim\left(e^{h_{\text {top }}(f)}\right)^{n}$. That is: the number of different orbits of length $n, x_{0}, x_{1}, \ldots, x_{n} ; x_{i+1}=f\left(x_{i}\right)$ such that $x_{j} \in A_{i_{j}}, j=0,1, \ldots, n-1$ is approximately equal to $\left(e^{h_{t o p}(f)}\right)^{n}$.

So, the greater entropy the map $f$ has then the greater number of different orbits the map has. Hence, to compute the topological entropy defined by a map $f: X \rightarrow X$ is an interesting problem.

The present paper is related with the computation of the topological entropy associated to certain subshifts of the shift map $\sigma: \Sigma_{2} \rightarrow \Sigma_{2}$ (here $\left.\Sigma_{2}=\{\theta: N \cup\{0\} \rightarrow\{0,1\}\}\right)$ and $\sigma\left(\theta_{0}, \theta_{1}, \theta_{2}, \ldots\right)=\left(\theta_{1}, \theta_{2}, \theta_{3}, \ldots\right)$. In fact, let us consider $a \in \mathcal{A}_{\infty}^{\infty}$ (see section 2 for the definition of the set $\mathcal{A}_{\infty}^{\infty}$ ) be a minimal periodic orbit. Accordingly with Aranzubia (see [4]) for $\Sigma\left[a_{-} \underline{b}, b_{+} \underline{a}\right]=\left\{\theta \in \Sigma_{2} ; a_{-} \underline{b} \leq \sigma^{i}(\theta) \leq b_{+} \underline{a}, \forall i \in N_{0}\right\}$ and $\left.\sigma\right|_{\Sigma\left[a_{-} \underline{b}, b_{+} \underline{a}\right]}$ : $\Sigma\left[a_{-} \underline{b}, b_{+} \underline{a}\right] \rightarrow \Sigma\left[a_{-} \underline{b}, b_{+} \underline{a}\right]$, we have:

$$
\begin{gathered}
(*) h_{\text {top }}\left(\left.\sigma\right|_{\Sigma\left[a_{-}, \underline{b}, b_{+} \underline{a}\right]}\right)=\frac{1}{\#(a)} \ln (2) ; \\
\text { here } \#(a)=\operatorname{per}(a) \\
=\text { period of the periodic sequence } \underline{a} .
\end{gathered}
$$


In this paper we compute $h_{t o p}\left(\left.\sigma\right|_{\Sigma\left[a_{-} \underline{b}, b_{+} \underline{a}\right]}\right)$ for $a \in \mathcal{A}_{\infty}^{\infty}$ such that $\#(a)$ is a prime number such that $2 \leq \#(a) \leq 10.000$ and we verify, in these cases, that the equation $(*)$ is true.

\section{Preliminaries}

\subsection{General Theory}

It is well known that one of the purposes of the topological theory of Dynamical Systems is to find universal models describing the topological dynamics of a large class of systems (see for instance [3], [9], [33]).

One of these universal models is the shift on $n$-symbols $\sigma: \Sigma_{n} \rightarrow \Sigma_{n}$ where $\Sigma_{n}$ is the set of sequences $\left\{\theta: N_{0} \rightarrow\{0,1,2, \ldots, n-1\}\right\}$ and $\sigma$ is the shift map defined by $(\sigma(\theta))(i)=\theta(i+1)$. Here $\Sigma_{n}$ is endowed with a certain topology and certain order.

In fact, several signed orders can be defined in $\Sigma_{n}$. Let us doing this here. Let $0=x_{0}<x_{1}<x_{2}<\ldots<x_{2 n-1}=1$ be $2 n$ points in the unit interval $[0,1]$. Let $I_{j}=\left[x_{2 j}, x_{2 j+1}\right]$ for $j=0,1,2, \ldots, n-1$; and $T: \bigcup_{j=0}^{n-1} I_{j} \rightarrow$ $[0,1]$ be a map such that its restriction to $I_{j}$ is linear and onto $[0,1]$, for any $j=0,1, \ldots, n-1$. The restriction of the map T to any interval $I_{j}$ can be either orientation preserving or orientation reversing. Hence, we may define $2^{n}$ piecewise linear maps $T: \bigcup_{j=0}^{n-1} I_{j} \rightarrow[0,1]$ as before. Let $\operatorname{Lin}(n)$ denote the set formed by these $2^{n}$ maps. Associated to any $T \in \operatorname{Lin}(n)$ we have its maximal invariant set $\Lambda_{T}=\left\{x \in \bigcup_{j=0}^{n-1} I_{j} ; T^{i}(x) \in \bigcup_{j=0}^{n-1} I_{j}\right.$, for all $\left.i \in N\right\}$. In this set, we consider the topology induced by the euclidean topology of the interval $[0,1]$.

It is not hard to see that the set $\Lambda_{T}$ is bijective to $\Sigma_{n}$. In fact, the itinerary map $I_{T}: \Lambda_{T} \rightarrow \Sigma_{n}$ defined by $I_{T}(x)(i)=j$ if and only if $T^{i}(x) \in$ $I_{j}$ is bijective. Its inverse map $I_{T}^{-1}: \Sigma_{n} \rightarrow \Lambda_{T}$ is given by $I_{T}^{-1}(\theta)=$ $I_{\theta_{0}} \cap T^{-1}\left(I_{\theta_{1}}\right) \cap T^{-2}\left(I_{\theta_{2}}\right) \cap \ldots=\bigcap_{j=0}^{\infty} T^{-j}\left(I_{\theta_{j}}\right)$, where, from now and on, we denote $\theta=\left(\theta_{0} \theta_{1} \theta_{2} \ldots\right)$. Hence, by using the bijective map $I_{T}: \Lambda_{T} \rightarrow \Sigma_{n}$ we can induce in $\Sigma_{n}$ :

(a) A topology $\tau_{T}: U \subset \Sigma_{n}$ is open if and only if $I_{T}^{-1}(U) \subset \Lambda_{T}$ is open, and 
(b) An order: $\theta \leq_{T} \beta$ in $\Sigma_{n}$ if and only if $I_{T}^{-1}(\theta) \leq I_{T}^{-1}(\beta)$ in $\Lambda_{T}$.

Let us denote by $\Sigma_{n}(T)$ the ordered, compact topological space $\left(\Sigma_{n}, \tau_{T}, \leq_{T}\right)$. In this way, we have introduced $2^{n}$ of these ordered compact metric spaces.

These models has been extensively used to obtain a great amount of information about maps defined in an interval (see for instances $[3,8,9$, $14,17,18,19,20,29,25])$; vector fields on three dimensional manifolds (see for instance $[2,7,12,13,15,21,22,32,34]$ ) among other kinds of dynamical systems.

In the special case of one dimensional dynamics, the shift of two symbols may be used to study increasing (decreasing) map with one discontinuity like the Lorenz maps, unimodal maps like the quadratic family or increasing-decreasing (decreasing-increasing) maps with one discontinuity. Namely, for $n=2$ the ordered metric compact space $\left(\Sigma_{2}, \tau_{T}, \leq_{T}\right)$ corresponding to the increasing-increasing map $\mathrm{T}$ is known as the lexicographical space which generates the lexicographical world (see for instance $[17,18,19,20,23,24])$ which is denoted LW.

In this work we deal with the lexicographical world. That is, here we consider the set $\Sigma_{2}$ with the topology induced by the map $T: I_{0} \cup I_{1} \rightarrow[0,1]$ such that $\left.T\right|_{I_{0}}$ and $\left.T\right|_{I_{1}}$ are increasing maps.

Let $\sigma: \Sigma_{2} \rightarrow \Sigma_{2}$ be the shift map $\sigma\left(\theta_{0}, \theta_{1}, \theta_{2}, \ldots\right)=\left(\theta_{1}, \theta_{2}, \ldots\right)$. Let $\Sigma_{0}$ and $\Sigma_{1}$ denote the sets $\left\{\theta \in \Sigma_{2} ; \theta_{0}=0\right\}$ and $\left\{\theta \in \Sigma_{2} ; \theta_{0}=\right.$ $1\}$ respectively. It is clear that $\Sigma_{2}=\Sigma_{0} \cup \Sigma_{1}$.

In $\Sigma_{2}$ the order induced by $T, \leq_{T}$, is the lexicographical order: $\theta<\alpha$ for any $\theta \in \Sigma_{0}$ and $\alpha \in \Sigma_{1}$ or $\theta<\alpha$ if there is $\overline{n \in N \text { such that } \theta_{i}}=\alpha_{i}$ for $i=0,1,2, \ldots, n-1$ and $\theta_{n}=0$ and $\alpha_{n}=1$.

For $a \leq b$ in $\Sigma_{2}$ let $[a, b]$ denote the interval $\left\{\theta \in \Sigma_{2} \mid a \leq \theta \leq\right.$ $b\} . \quad \Sigma[a, b]$ will denote the invariant set $\bigcap_{n=0}^{\infty} \sigma^{-n}([a, b])$.

Let $a$ denote the finite string $a=a_{0} a_{1} \ldots a_{n}$ and $\underline{a}$ be the infinite sequence $\underline{a}=a_{0} a_{1} \ldots a_{n}=a_{0} a_{1} \ldots a_{n}, a_{0} a_{1} \ldots a_{n}, \ldots$ For example, if $a=$ 0011 then $\underline{a}=\underline{0011}=00110011001100110011 \ldots$

Let $\operatorname{Max}_{2}=\left\{\theta \in \Sigma_{2} ; \sigma^{i}(\theta) \leq \theta, \forall i \in N_{0}\right\}$ and $\operatorname{Min}_{2}=\left\{\alpha \in \Sigma_{2} ; \alpha \leq\right.$ $\left.\sigma^{i}(\alpha), \forall i \in N_{0}\right\}$ denotes the sets of maximal and minimal sequences in the lexicographical order.

Definition 2.1. The set $L W=\left\{(a, b) \in \operatorname{Min}_{2} \times \operatorname{Max}_{2} ;\{a, b\} \subset \Sigma[a, b]\right\}$ will be called the lexicographical world. 
The lexicographical world may be used to model the standard two parameter family of Lorenz maps given by

$$
F_{(\mu, \nu)}(x)=\left\{\begin{array}{ll}
-x^{2}+\nu & , \quad x<0 \\
x^{2}-\mu & , \quad x>0
\end{array} \quad \mu, \nu \geq 0\right.
$$

In fact, let us consider the restriction of the dynamics of the map $F_{(\mu, \nu)}$ to the interval $I(\mu, \nu)=[-\mu, \nu]$. That is $F_{(\mu, \nu)}: I(\mu, \nu) \rightarrow I(\mu, \nu)$. Let $\Lambda(\mu, \nu)=\bigcap_{j=0}^{\infty} F_{(\mu, \nu)}^{-j}(I(\mu, \nu) \backslash\{0\})$. For any $x \in \Lambda(\mu, \nu)$ let us consider its itinerary $I(\mu, \nu)(x) \in \Sigma_{2}=\left\{\theta: N_{0} \rightarrow\{0,1\}\right\}$ given by $I(\mu, \nu)(x)(i)=$ $j \Leftrightarrow F_{(\mu, \nu)}^{i}(x) \in I_{j}(\mu, \nu)$; where $I_{0}(\mu, \nu)=\{x \in \Lambda(\mu, \nu) ; x<0\}$ and $I_{1}(\mu, \nu)=\{x \in \Lambda(\mu, \nu) ; x>0\}$.

$I(\mu, \nu): \Lambda(\mu, \nu) \rightarrow \Sigma_{2}$ is a continuous map whose image $I(\mu, \nu)(\Lambda(\mu, \nu))=$ $\{I(\mu, \nu)(x) ; x \in \Lambda(\mu, \nu)\}=\Sigma[\mu, \nu]$ satisfies $\overline{\Sigma[\mu, \nu]}=\bigcap_{n=0}^{\infty} \sigma^{-n}([a(\mu, \nu), b(\mu, \nu)])$ where $a(\mu, \nu)=\lim _{x \downarrow-\mu} I(\mu, \nu)(x)$ and $b(\mu, \nu)=\lim _{x \uparrow \nu} I(\mu, \nu)(x)$; here the limit is taken over elements $x \in \Lambda(\mu, \nu)$. The sequences $a(\mu, \nu)$ and $b(\mu, \nu)$ satisfies $\sigma^{i}(a(\mu, \nu)) \geq a(\mu, \nu)$ and $\sigma^{i}(b(\mu, \nu)) \leq b(\mu, \nu)$ for any $i \in N_{0}$.

Now we can consider the map $\alpha:\{(\mu, \nu) ; \mu \geq 0, \nu \geq 0\} \rightarrow$ Min $_{2}$ and $\beta:\{(\mu, \nu) ; \mu \geq 0, \nu \geq 0\} \rightarrow M_{a x}$ defined by $\alpha(\mu, \nu)=a(\mu, \nu)$ and $\beta(\mu, \nu)=b(\mu, \nu)$, which associate to any $(\mu, \nu)$ its kneading sequences $a(\mu, \nu)$ and $b(\mu, \nu)$. These maps are not continuous (see [20]).

It is clear that we can parameterize the positive $(\mu, \nu)$-plane by using the map $\alpha$ or the map $\beta$. In this way we have an $\alpha$ and a $\beta$-decomposition of the positive $(\mu, \nu)$-plane.

Also, we have a map $L:\{(\mu, \nu) ; \mu \geq 0, \nu \geq 0\} \rightarrow L W$ given by $L(\mu, \nu)=$ $(\alpha(\mu, \nu), \beta(\mu, \nu))=(a(\mu, \nu), b(\mu, \nu))$. This map is not continuous (see [20]). Let us consider $h_{\text {top }}:\{(\mu, \nu) ; \mu \geq 0, \nu \geq 0\} \rightarrow[0, \ln (2)]$ the topological entropy $h_{\text {top }}(\mu, \nu)=h_{\text {top }}\left(\left.\sigma\right|_{\Sigma[a(\mu, \nu), b(\mu, \nu)]}\right)$. It is unknown if this map is continuous or not. Also, it is unknown if the set $\left\{(\mu, \nu): h_{t o p}(\mu, \nu)=0\right\}$ is a connected set. For us this set (the entropy zero set) seems to be arc connected but not locally connected.

Observation 2.2. Let us now establish some notations.

1.- If $\underline{a} \in \operatorname{Min}_{2}, \underline{a} \neq \underline{0}$ then $\underline{b(a)}=\sup \left\{\sigma^{i}(\underline{a}) ; i \in N\right\} \in \operatorname{Max}_{2}$ and if $\underline{d} \in \operatorname{Max}_{2}, \underline{d} \neq \underline{1}$ then $\overline{a(d)}=\inf \left\{\sigma^{i}(\underline{d}) ; i \in N\right\} \in$ Min $_{2}$, if $\underline{b(a)}=\underline{b_{1} b_{2} \ldots b_{n}}$ then we will denote $b(a)=b_{1} b_{2} \ldots b_{n}$. 
2.- If $a=0 a_{1} a_{2} \ldots a_{n-1} 1$ then $a_{-}=0 a_{1} a_{2} \ldots a_{n-1} 0$ and if $b=1 b_{1} b_{2} \ldots b_{n-1} 0$ then $b_{+}=1 b_{1} b_{2} \ldots b_{n-1} 1$.

3.- If $\underline{a_{1}}, \underline{a_{2}}$ are two sequences then we define $m\left(a_{1}, a_{2}\right)$ by $m\left(a_{1}, a_{2}\right)=$ $\underline{a_{1}} \overline{a_{2}}$. For instance for $\underline{a_{1}}=\underline{001}$ and $\underline{a_{2}}=\underline{01}$ we have $m\left(a_{1}, a_{2}\right)=$ $\underline{00101}$.

\subsection{The set $\mathcal{A}_{\infty}^{\infty}$}

Let us define the set $\mathcal{A}_{\infty}^{\infty}$

Definition 2.3. Let $A_{0}=\left\{\underline{0^{n} 1}, \underline{01^{m}} ; n, m \in N\right\}$, that is:

$$
A_{0}=\{\ldots \underline{00001}, \underline{0001}, \underline{001}, \underline{01}, \underline{011}, \underline{0111}, \underline{01111 \ldots\}} .
$$

Let $A_{1}=\left\{m\left(a_{1}, a_{2}\right), \underline{a_{1}}<\underline{a_{2}}\right.$ are consecutive sequences in $\left.A_{0}\right\} \cup A_{0}$ and $A_{n+1}=\left\{m\left(a_{1}, a_{2}\right) ; \underline{a_{1}}<\underline{a_{2}}\right.$ are consecutive sequences in $\left.A_{n}\right\} \cup A_{n}$. So, we have:

$$
\begin{gathered}
A_{0}=\ldots \underline{00001}, \underline{0001}, \underline{001}, \underline{01}, \underline{011}, \underline{0111}, \frac{01111}{\ldots} \ldots \\
A_{1}=\ldots \underline{0001}, \frac{0001001}{\downarrow}, \underline{001}, \frac{00101}{\downarrow}, \underline{01}, \frac{01011}{\downarrow}, \underline{011}, \frac{0110111}{\downarrow}, \underline{0111} \ldots \\
m(\underline{0001}, \underline{001}) \\
m(\underline{001}, \underline{01}) \\
m(\underline{01}, \underline{011})
\end{gathered}
$$

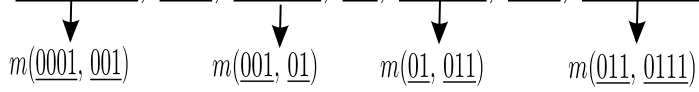

$A_{2}=\ldots \underline{001}, \underline{00100101}, \underline{00101}, \underline{0010101}, \underline{01}, \underline{0101011}, \underline{01011}, \underline{01011011}, \underline{011} \ldots$

Let $\mathcal{A}_{\infty}=\bigcup_{n=0}^{\infty} A_{n}$. For $\underline{a} \in \mathcal{A}_{\infty}$ let

$A_{0}(\underline{a})=\left\{a_{-}(b(a))^{m-1} b(a)_{+}, a_{-} b(a)_{+} a^{n-1} ; n, m \in N\right\} ;$

$A_{1}(\underline{a})=\left\{m\left(a_{1}, a_{2}\right) ; \underline{a_{1}}<\underline{a_{2}}\right.$ are consecutive sequences in $\left.A_{0}(\underline{a})\right\} \cup A_{0}(\underline{a})$; and

$A_{n+1}(\underline{a})=\left\{m\left(a_{1}, a_{2}\right) ; \underline{a_{1}}<\underline{a_{2}}\right.$ are consecutive sequences in $\left.A_{n}(\underline{a})\right\} \cup A_{n}(\underline{a})$

and let $\mathcal{A}_{\infty}(\underline{a})=\bigcup_{n=0}^{\infty} A_{n}(\underline{a})$.

Example: For $a=\underline{01} \in \mathcal{A}_{\infty}$, we have:

$A_{0}(a)=\{\ldots \underline{00101011}, \underline{001011}, \underline{0011}, \underline{001101}, \underline{00110101}, \ldots\}$

$A_{1}(a)=\{\ldots \underline{001011}, \underline{0010110011}, \underline{0011}, \underline{0011001101}, \underline{001101}, \underline{00110100110101}, \ldots\}$.

Let us consider $\mathcal{A}_{\infty}^{0}=\mathcal{A}_{\infty}$ and $\mathcal{A}_{\infty}^{1}=\bigcup_{\underline{a} \in \mathcal{A}_{\infty}} \mathcal{A}_{\infty}(\underline{a}) \cup \mathcal{A}_{\infty}^{0}$.

Now, associated to any $\underline{a} \in \mathcal{A}_{\infty}^{1}$ we construct:

$A_{0}^{2}(\underline{a})=\left\{\underline{a_{-}(b(a))^{m-1}(b(a))_{+}}, \underline{a_{-} b(a)_{+} a^{n-1}} ; n, m \in N\right\} ;$ 

$A_{n}^{2}(\underline{a})$;

$A_{n+1}^{2}(\underline{a})=\left\{m\left(a_{1}, a_{2}\right) ; \underline{a_{1}}<\underline{a_{2}}\right.$ are consecutive sequences in $\left.A_{n}^{2}(\underline{a})\right\} \cup$

$\mathcal{A}_{\infty}^{2}(\underline{a})=\bigcup_{n=0}^{\infty} A_{n}^{2}(\underline{a})$ and $\mathcal{A}_{\infty}^{2}=\bigcup_{\underline{a} \in \mathcal{A}_{\infty}^{1}} \mathcal{A}_{\infty}^{2}(\underline{a}) \cup \mathcal{A}_{\infty}^{1}$.

Similarly, for any $n \geq 2$, we may define: $\mathcal{A}_{\infty}^{n+1}$ and $\mathcal{A}_{\infty}^{\infty}=\bigcup_{n=0}^{\infty} \mathcal{A}_{\infty}^{n}$.

Observation 2.4. $\quad$ - We note that all the elements in $\mathcal{A}_{\infty}^{\infty}$ are minimal periodic sequences.

- There are minimal periodic sequences $\underline{a} \in \operatorname{Min}_{2}$ such that $\underline{a} \notin \mathcal{A}_{\infty}^{\infty}$. For instance: $\underline{a}=\underline{00111}, \underline{a}=\underline{000111}$ and $\underline{a}=\underline{001011011}$.

As part of his PH.D. Thesis Solange Aranzubia (see [4]) proved the following:

Theorem 2.5. For any $a \in \mathcal{A}_{\infty}^{\infty}$ we have that, $h_{\text {top }}\left(a_{-} \underline{b}, b_{+} \underline{a}\right)=\frac{1}{\operatorname{Per}(a)} \ln (2)$.

So, from this result we conclude that: For elements in $\mathcal{A}_{\infty}^{\infty}$ the longer the period of the periodic sequence is then the lower complexity in the dynamics of the extension the associated map has.

This fact is not true for any minimal periodic sequence $\underline{a} \in M i n_{2}$. In fact,

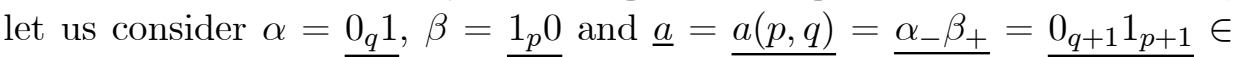
$\mathrm{Min}_{2}$. For this sequence and for $\underline{b}=\underline{b(a)}=\underline{\underline{b(p, q)}}=\underline{1_{p+1} 0_{q+1}}$ we have that

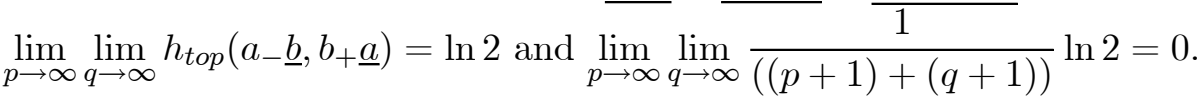

In the present paper we compute the value of the topological entropy, for all the periodic sequences $a \in \mathcal{A}_{\infty}^{\infty}$ whose period is a prime number, $p$, between 3 and 9973, by using an algorithm associated to graphs. We also verify that the cost of the algorithm is $\mathcal{O}\left(p^{3}\right)$. These computations can be considered as a computer verification of this theorem for prime period in this range.

\subsection{Computation of the topological entropy for the map}

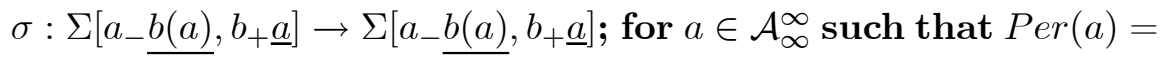
$p$ is a prime number.

Initially, let us consider $a_{p}=\underline{0_{p-1} 1}=\underbrace{0 \ldots 0}_{p-1} 1 \underbrace{0 \ldots 0}_{p-1} 1 \ldots$ the sequence of period $p$ which has $(p-1)$-zeroes and one one.

Associated to $a_{p}$ we will construct a discontinuous map $f:[0,1] \backslash\left\{c_{p}\right\} \rightarrow$ $[0,1]$ that satisfies: 
i) For $\alpha_{0}=0$ we have $I_{f}\left(\alpha_{0}\right)=\left(a_{p}\right)_{-} \underline{b\left(a_{p}\right)}$

ii) For $\beta=1$ we have $I_{f}(\beta)=\left(b\left(a_{p}\right)\right)+\underline{a_{p}}$

iii) $\left.f\right|_{\left[\alpha_{0}, c_{p}\right]}$ is increasing and continuous and $f\left(c_{p}^{-}\right)=\beta$

iv) $\left.f\right|_{\left[c_{p}, \beta\right]}$ is increasing and continuous and $f\left(c_{p}^{+}\right)=\alpha_{0}$

To construct the map $f$ let us consider numbers $\alpha_{0}=0<\alpha_{1}<\alpha_{2}<$ $\ldots<\alpha_{p-1}<c_{p}<\alpha_{p}<\beta=1$.

For the map $f$ we have: $f\left(\alpha_{0}\right)=\alpha_{1}, f\left(\alpha_{1}\right)=\alpha_{2}, \ldots, f\left(\alpha_{p-1}\right)=$ $\alpha_{p}, f\left(c_{p}\right)=1, f\left(\alpha_{p}\right)=\alpha_{1}, f(\beta)=\alpha_{2}$ and, in between two of these numbers, the map $\mathrm{f}$ is linear. We have figure 1 :

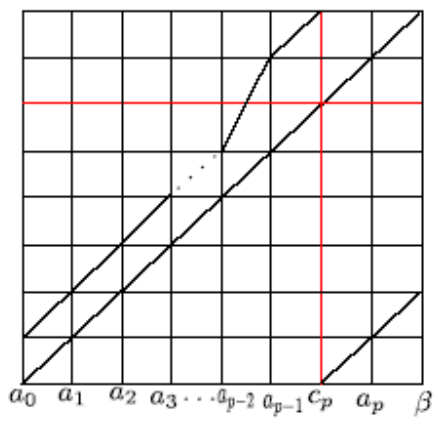

Figure 1: Function $f$.

Concerning the itineraries associated to these points we have $I_{f}\left(\alpha_{0}\right)=$ $0_{p} 10_{p-1} 1, I_{f}\left(\alpha_{1}\right)=0_{p-1} 1, I_{f}\left(\alpha_{2}\right)=0_{p-2} 10, I_{f}\left(\alpha_{3}\right)=0_{p-3} 100, \ldots, I_{f}\left(\alpha_{p-1}\right)=$

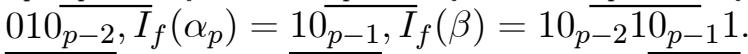

Now, let us denote by $I_{1}=\left[\alpha_{p-2}, \alpha_{p-1}\right], I_{2}=\left[\alpha_{p-1}, c_{p}\right], I_{3}=\left[c_{p}, \alpha_{p}\right], I_{4}=$ $\left[\alpha_{p}, \beta\right], I_{5}=\left[\alpha_{0}, \alpha_{1}\right], I_{6}=\left[\alpha_{1}, \alpha_{2}\right], I_{7}=\left[\alpha_{2}, \alpha_{3}\right], \ldots, I_{p+2}=\left[\alpha_{p-1}, \alpha_{p-2}\right]$.

The map $f$ satisfies: $f\left(I_{1}\right)=I_{2} \cup I_{3}, f\left(I_{2}\right)=I_{4}, f\left(I_{3}\right)=I_{5}, f\left(I_{4}\right)=$ $I_{6}, f\left(I_{5}\right)=I_{6}, f\left(I_{6}\right)=I_{7}, f\left(I_{7}\right)=I_{8} \ldots, f\left(I_{p+2}\right)=I_{1}$. Hence we have the $A$-graph (Figure 2) see [4] or [16]. 


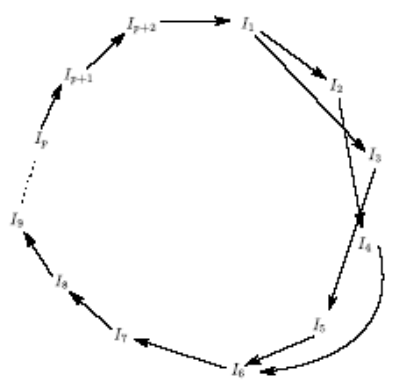

Figure 2: A-graph

Associated to these graph we have the incidence matrix $M$ given by

$$
M=\left(\begin{array}{llllllllll}
0 & 1 & 1 & 0 & 0 & 0 & 0 & 0 & \cdots & 0 \\
0 & 0 & 0 & 1 & 0 & 0 & 0 & 0 & \cdots & 0 \\
0 & 0 & 0 & 0 & 1 & 0 & 0 & 0 & \cdots & 0 \\
0 & 0 & 0 & 0 & 0 & 1 & 0 & 0 & \cdots & 0 \\
0 & 0 & 0 & 0 & 0 & 1 & 0 & 0 & \cdots & 0 \\
0 & 0 & 0 & 0 & 0 & 0 & 1 & 0 & \cdots & 0 \\
& & & & \vdots & & & & & \\
0 & 0 & 0 & 0 & 0 & 0 & 0 & 0 & \cdots & 1 \\
1 & 0 & 0 & 0 & 0 & 0 & 0 & 0 & \cdots & 0
\end{array}\right)
$$

This matrix has $\lambda=0$ as eigenvalue and $h_{t o p}\left(\left.\sigma\right|_{\Sigma\left[0_{p} 10_{p-1} 1,10_{p-2} 10_{p-1} 1\right]}\right)=$

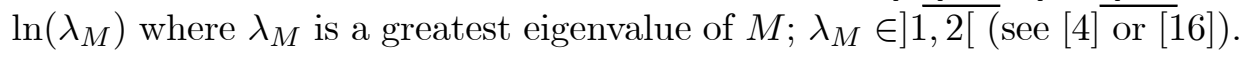

Now, by using $a_{p}=0_{p-1} 1$ and an algorithm, we are able to construct $p-2$ sequences $a_{1}, a_{2}, \cdots, a_{p-2}$ such that period $\left(a_{i}\right)=p$ and $a_{i} \in \mathcal{A}_{\infty}^{\infty}$, for any $i=1,2, \cdots, p-2$.

Accordingly with Theorem 3.1.5 in [4], for any other minimal sequence $d \in \Sigma_{0}$ such that $\operatorname{period}(d)=p$ we have that $d \notin \mathcal{A}_{\infty}^{\infty}$.

Hence, our algorithm give us all the minimal periodic sequences $a$ with $\operatorname{period}(a)=p$ and $a \in \mathcal{A}_{\infty}^{\infty}$.

The general construction of the algorithm may be found in section 3.1.1 in [4], pages 57-76, and will appear elsewhere.

Here, for the sake of completeness, we will show the algorithm for the cases $\operatorname{period}(a)=3,5$, and 7 .

Let us begin with the case $p=3$.

For $a=\underline{001}, b=\underline{100}$, we have $a_{-} \underline{b}=000 \underline{100}=0 \underline{001}$ and $b_{+} \underline{a}=101 \underline{001}=$ $1 \underline{010}$, so $\overline{a_{-} \underline{b}}=\underline{0 \underline{001}}=0 \rightarrow \sigma(\underline{0001})=\underline{001}=1 \rightarrow \sigma(\underline{001})=\underline{010}=2 \rightarrow$ 
$\sigma(\underline{010})=\underline{100}=3 \rightarrow \sigma(\underline{100})=\underline{001}=1$;

$b_{+} \underline{a}=1 \underline{010}=4 \rightarrow \sigma(\underline{1010})=\underline{010}=2$.

Now we construct a permutation associated the iteration (under $\sigma$ ) of the elements

$a_{-} \underline{b}=000 \underline{100}=0 \underline{001}$ and $b_{+} \underline{a}=101 \underline{001}=1 \underline{010}$,

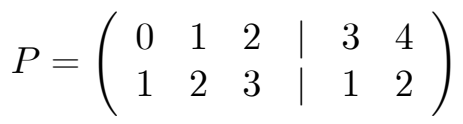

This permutation allows us to define a discontinuous map of the interval (like the previous $f$ ).

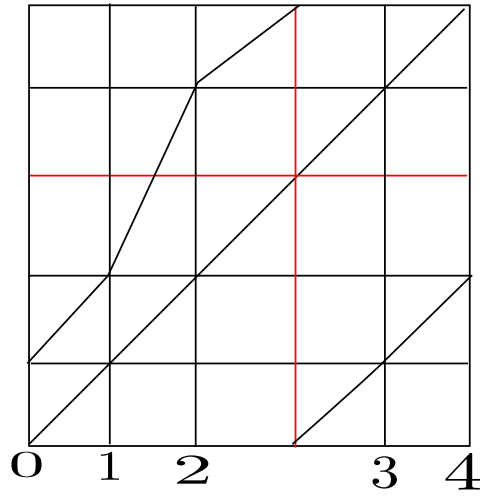

This map represents the dynamics of $\left.\sigma\right|_{\left[a_{-} \underline{b}, b_{+} \underline{a}\right]}$ where $a_{-} \underline{b}=0 \underline{001}$ and $b_{+} \underline{a}=1 \underline{010}$

Now, multiplying the permutation $P$ by itself we have $P \cdot P$

$$
\begin{gathered}
P \cdot P=\left(\begin{array}{llllll}
0 & 1 & 2 & \mid & 3 & 4 \\
1 & 2 & 3 & \mid & 1 & 2
\end{array}\right) \cdot\left(\begin{array}{llllll}
0 & 1 & 2 & \mid & 3 & 4 \\
1 & 2 & 3 & \mid & 1 & 2
\end{array}\right) \\
=\left(\begin{array}{llllll}
0 & 1 & 2 & 3 & 4 \\
2 & 3 & \mid & 1 & 2 & 3
\end{array}\right)=P^{2}
\end{gathered}
$$

Using $P^{2}$ we can define a discontinuous map of the interval: 


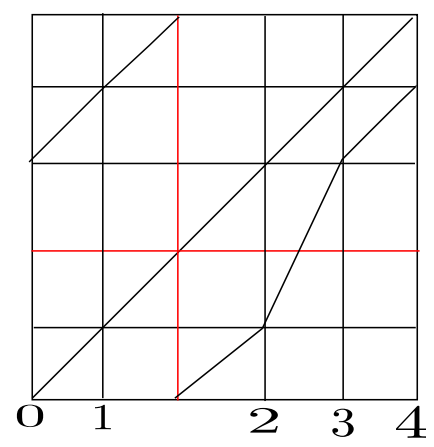

This map represent the dynamics of $\left.\sigma\right|_{\left[a_{-} \underline{b}, b_{+} \underline{a}\right]}$ where $a_{-} \underline{b}=01 \underline{011}$ and $b_{+} \underline{a}=1 \underline{110}$, therefore, for the sequence $a$, we have: $a=\underline{011}$ and $\operatorname{Per}(a)=3$.

Let us now consider the multiplication $P \cdot P^{2}$, we have:

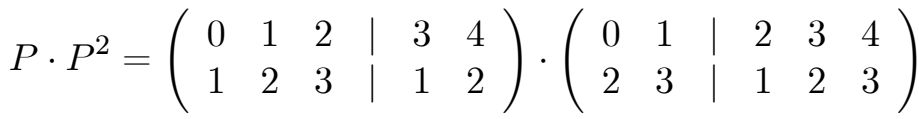

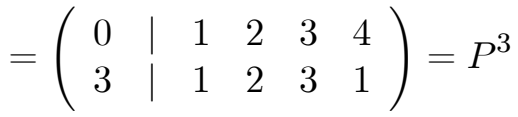

Using this permutation we cannot construct a dynamics that represents some subshift of the lexicographical world.

As we known, there are only two minimal periodic orbits of period three and both of them are in $\mathcal{A}_{\infty}^{\infty}$. These sequences are: $\underline{001}$ and $\underline{011}$. So, the proof in the case $p=3$ is complete.

- Let us work now with the case $p=5$.

Let $a=\underline{00001}, b=\underline{10000}$, then $a_{-} \underline{b}=00000 \underline{10000}=0 \underline{00001}$ and $b_{+} \underline{a}=$ $10001 \underline{00001}=1 \underline{00010}$. Therefore:

$a_{-} \underline{b}=\underline{000001}=0 \rightarrow \sigma(\underline{000001})=\underline{00001}=1 \rightarrow \sigma(\underline{00001})=\underline{00010}=2 \rightarrow$ $\sigma(\underline{00010})=\underline{00100}=3 \rightarrow \sigma(\underline{00100})=\underline{01000}=4 \rightarrow \sigma(\underline{01000})=\underline{10000}=$ $5 \rightarrow \sigma(\underline{10000})=\underline{00001}=1$;

$b_{+} \underline{a}=1 \underline{00010}=6 \rightarrow \sigma(\underline{100010})=\underline{00010}=2$.

So, associated to the iterations (by $\sigma$ ) of the sequences $a_{-} \underline{b}$ and $b_{+} \underline{a}$ we have the following permutation:

$$
P=\left(\begin{array}{lllll|ll}
0 & 1 & 2 & 3 & 4 & 5 & 6 \\
1 & 2 & 3 & 4 & 5 & 1 & 2
\end{array}\right)
$$

This permutation allows us to define a discontinuous map of the interval (like in the previous case $p=3$ ) 


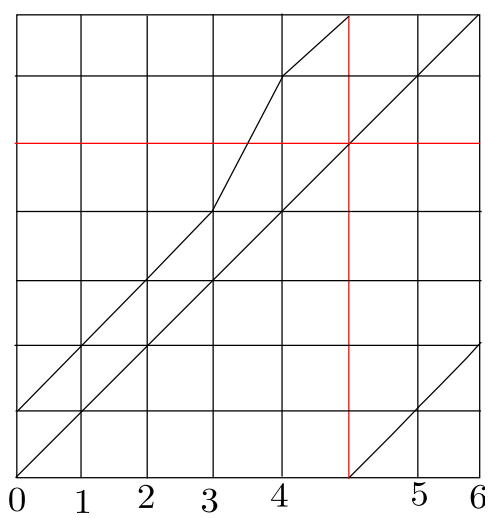

This map represent the dynamics of $\left.\sigma\right|_{\left[a_{-}, \underline{b}, b_{+} \underline{a}\right]}$, where $a_{-} \underline{b}=0 \underline{00001}$ and $b_{+} \underline{a}=1 \underline{00010}$. Therefore, for the sequence $a$, we have: $a=\underline{00001}$ and $\operatorname{Per}(a)=5$.

Now, let us compute $P \cdot P$

$$
\begin{gathered}
P \cdot P=\left(\begin{array}{llllllll}
0 & 1 & 2 & 3 & 4 & \mid & 5 & 6 \\
1 & 2 & 3 & 4 & 5 & \mid & 1 & 2
\end{array}\right) \cdot\left(\begin{array}{cccccccc}
0 & 1 & 2 & 3 & 4 & a & 5 & 6 \\
1 & 2 & 3 & 4 & 5 & a & 1 & 2
\end{array}\right) \\
=\left(\begin{array}{llllllll}
0 & 1 & 2 & 3 & \mid & 4 & 5 & 6 \\
2 & 3 & 4 & 5 & 1 & 2 & 3
\end{array}\right)=P^{2}
\end{gathered}
$$

Using $P^{2}$ we can define a discontinuous map of the interval:

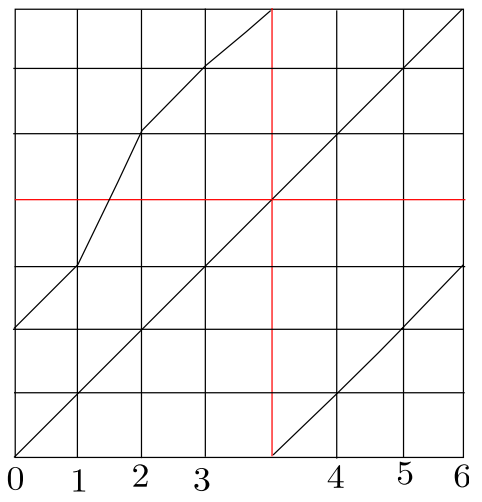

This map represent the dynamics of $\left.\sigma\right|_{\left[a_{-} \underline{b}, b_{+} \underline{a}\right]}$, with $a_{-} \underline{b}=0 \underline{01001}$ and $b_{+} \underline{a}=1 \underline{01010}$, where $a=\underline{00101}$ and $\operatorname{per}(a)=5$.

Now, let us continuous with $P^{3}$ : 


$$
\begin{gathered}
P \cdot P^{2}=\left(\begin{array}{cccccccc}
0 & 1 & 2 & 3 & 4 & \mid & 5 & 6 \\
1 & 2 & 3 & 4 & 5 & \mid & 1 & 2
\end{array}\right) \cdot\left(\begin{array}{llll|lll}
0 & 1 & 2 & 3 & 4 & 5 & 6 \\
2 & 3 & 4 & 5 & 1 & 2 & 3
\end{array}\right) \\
=\left(\begin{array}{llllllll}
0 & 1 & 2 & 3 & 4 & 5 & 6 \\
3 & 4 & 5 & \mid & 1 & 2 & 3 & 4
\end{array}\right)=P^{3}
\end{gathered}
$$

Using $P^{3}$ we can define a discontinuous map of the interval:

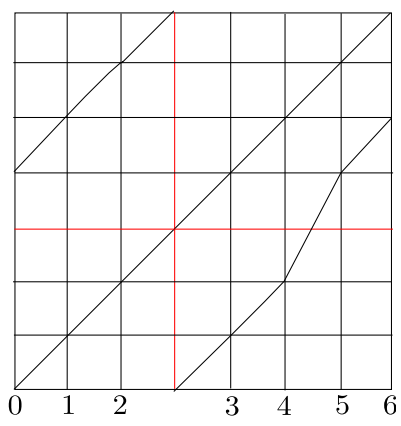

This map represent the dynamics of $\left.\sigma\right|_{\left[a_{-}, \underline{b} b_{+} \underline{a}\right]}$ with $a_{-} \underline{b}=01 \underline{01011}$ and $b_{+} \underline{a}=1 \underline{10110}$, where $a=\underline{01011}$ and $\operatorname{Per}(a)=5$.

Now, let us continuous with $P^{4}$ :

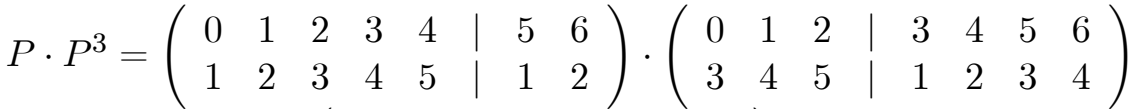

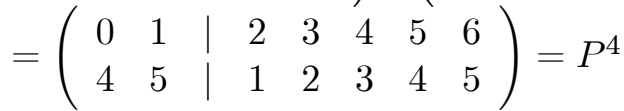

Using $P^{4}$ we can define a discontinuous map of the interval

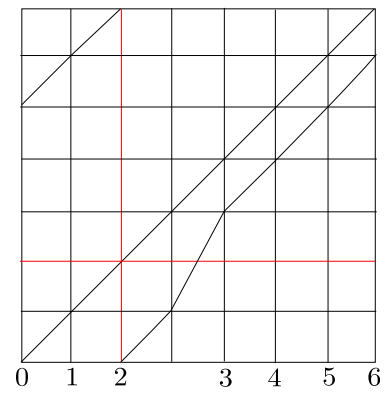

This map represent the dynamics of $a_{-} \underline{b}=0 \underline{11101}$ and $b_{+} \underline{a}=1 \underline{11110}$, 
where $a=\underline{01111}$ and $\operatorname{Per}(a)=5$.

Now, for $P^{5}$ we have:

$$
\begin{gathered}
P \cdot P^{4}=\left(\begin{array}{cccccccc}
0 & 1 & 2 & 3 & 4 & \mid & 5 & 6 \\
1 & 2 & 3 & 4 & 5 & \mid & 1 & 2
\end{array}\right) \cdot\left(\begin{array}{llllllll}
0 & 1 & 2 & 3 & 4 & 5 & 6 \\
4 & 5 & \mid & 2 & 3 & 4 & 5
\end{array}\right) \\
=\left(\begin{array}{llllllll}
0 & \mid & 1 & 2 & 3 & 4 & 5 & 6 \\
5 & \mid & 1 & 2 & 3 & 4 & 5 & 1
\end{array}\right)=P^{5}
\end{gathered}
$$

Using this permutation we cannot construct a map whose dynamics represents some subshift of the lexicographical world.

Now, these four sequences are minimal periodic sequences with period 5 in $\mathcal{A}_{\infty}^{\infty}$. These sequences are: $\underline{00001}, \underline{00101}, \underline{01011}$ and $\underline{01111}$.

There are another two minimal periodic sequences of period 5. These are: $\underline{00111}=\underline{a_{-} d_{+}}$with $a=\underline{01}, d=\underline{110}$ and $\underline{00011}=\underline{a_{-} d_{+}}$with $a=$ $\underline{001}, d=\underline{10}$.

Accordingly with section 3.1.1 in [4] we have $h_{\text {top }}(\underline{00111}, \underline{11100})=h_{\text {top }}(\underline{00011}, \underline{11000})=h_{\text {top }}(\underline{01}, \underline{110})=h_{\text {top }}(\underline{001}, \underline{10})>$ 0 .

We observe, accordingly with section 3.1.1. in [4] that for any $a \in \mathcal{A}_{\infty}^{\infty}$ we have that $h_{t o p}(a, b(a))=0$. Hence, we conclude that $\underline{00111}$ and $\underline{00011}$ are minimal periodic sequences not in $\mathcal{A}_{\infty}^{\infty}$.

- Let us now consider minimal periodic sequences with period $p=7$. Let $a=\underline{0000001}=\underline{0_{6} 1}, b=\underline{1000000}=\underline{10_{6}}$. We have: $a_{-} \underline{b}=\underline{00_{6} 1}$ and $b_{+} \underline{a}=10_{5} 10$. Hence:

$a_{-} \underline{b}=0 \overline{0_{6} 1}=0 \rightarrow \sigma\left(00_{6} 1\right)=\underline{0_{6} 1}=1 \rightarrow \sigma\left(\underline{\left.0_{6} 1\right)}=\underline{0_{5} 10}=2 \rightarrow \sigma\left(\underline{\left.0_{5} 10\right)}=\right.\right.$ $\underline{0_{4} 100}=\overline{3 \rightarrow \sigma}\left(\underline{0_{4} 100}\right)=\underline{0_{3} 10_{3}}=4 \rightarrow \sigma\left(\underline{\left.0_{3} 10_{3}\right)}=\underline{0_{2} \overline{10_{4}}}=5 \rightarrow \sigma\left(\underline{0_{2} 10_{4}}\right)=\right.$ $\underline{010_{5}}=6 \rightarrow \sigma\left(\underline{\left.010_{5}\right)}=10_{6}=7\right.$;

$\overline{b_{+} \underline{a}}=1 \underline{0_{5} 10}=\overline{8 \rightarrow} \sigma\left(\underline{10_{5} 10}\right)=\underline{0_{5} 10}=1$.

So, associated to the iterations (by $\sigma$ ) of the sequences $a_{-} \underline{b}$ and $b_{+} \underline{a}$ we have the following permutation:

$$
P=\left(\begin{array}{lllllll|ll}
0 & 1 & 2 & 3 & 4 & 5 & 6 & 7 & 8 \\
1 & 2 & 3 & 4 & 5 & 6 & 7 & 1 & 2
\end{array}\right)
$$

This permutation allows us to define a discontinuous map of the interval (like in the previous cases $p=3,5$ ) 


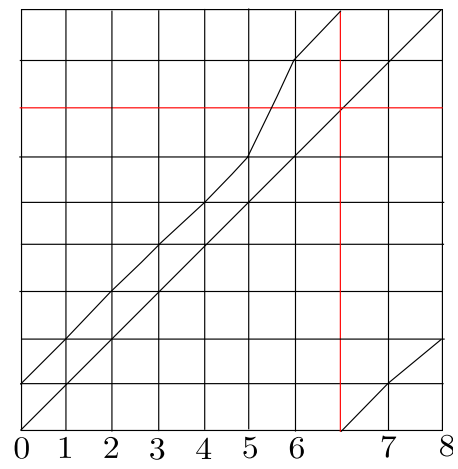

This map represent the dynamics of $\left.\sigma\right|_{\left[a_{-} \underline{b}, b_{+} \underline{a}\right]}$, with $a_{-} \underline{b}=0 \underline{00_{6} 1}$ and $b_{+} \underline{a}=10_{5} 10$, where $a=\underline{0000001}$ and $\operatorname{Per}(a)=7$.

Let us now consider $P^{2}$ :

$$
P^{2}=P \cdot P=\left(\begin{array}{llllllllll}
0 & 1 & 2 & 3 & 4 & 5 & \mid & 6 & 7 & 8 \\
2 & 3 & 4 & 5 & 6 & 7 & \mid & 2 & 2 & 3
\end{array}\right)
$$

This permutation allows us to define a discontinuous map of the interval (like in the previous case $p=3,5$ )

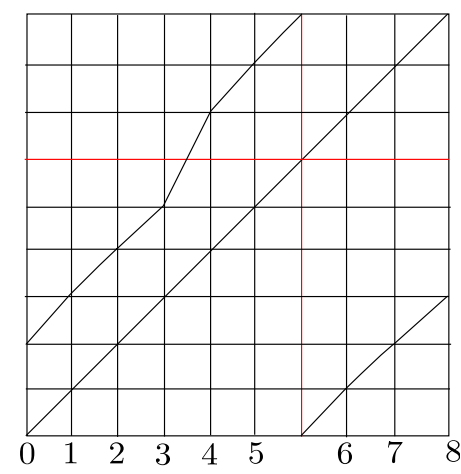

This map represent the dynamics of $\left.\sigma\right|_{\left[a_{-} \underline{b}, b_{+} \underline{a}\right]}$ with $a_{-} \underline{b}=0 \underline{0010001}$ and $b_{+} \underline{a}=1 \underline{0010010}$, where $a=\underline{0001001}$ and $\overline{\operatorname{Per}}(a)=7$.

Let us now consider $P^{3}$ :

$$
P^{3}=P \cdot P^{2}=\left(\begin{array}{ccccc|cccc}
0 & 1 & 2 & 3 & 4 & 5 & 6 & 7 & 8 \\
3 & 4 & 5 & 6 & 7 & 1 & 2 & 3 & 4
\end{array}\right)
$$

This permutation allows us to define a discontinuous map of the interval (like in the previous cases $p=3,5$ ) 


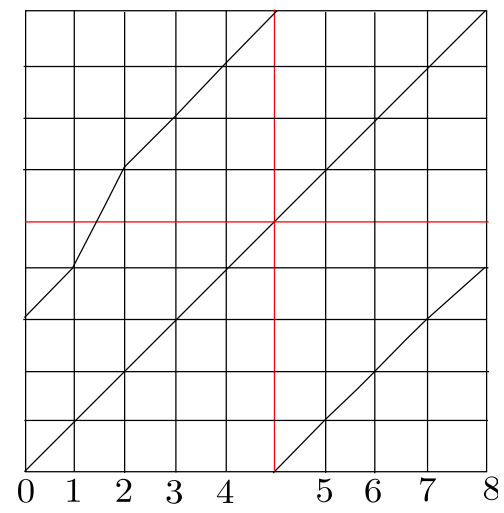

This map represents the dynamics of $\left.\sigma\right|_{\left[a_{-}, \underline{b}, b_{+} \underline{a}\right]}$ with $a_{-} \underline{b}=0 \underline{0101001}$ and $b_{+} \underline{a}=1 \underline{0101010}$, for $a=\underline{0010101}$ and $\operatorname{Per}(a)=7$.

Let us consider $P^{4}$ :

$$
P^{4}=P \cdot P^{3}=\left(\begin{array}{cccc|ccccc}
0 & 1 & 2 & 3 & 4 & 5 & 6 & 7 & 8 \\
4 & 5 & 6 & 7 & 1 & 2 & 3 & 4 & 5
\end{array}\right)
$$

This permutation allows us to define a discontinuous map of the interval (like in the previous cases $p=3,5$ )

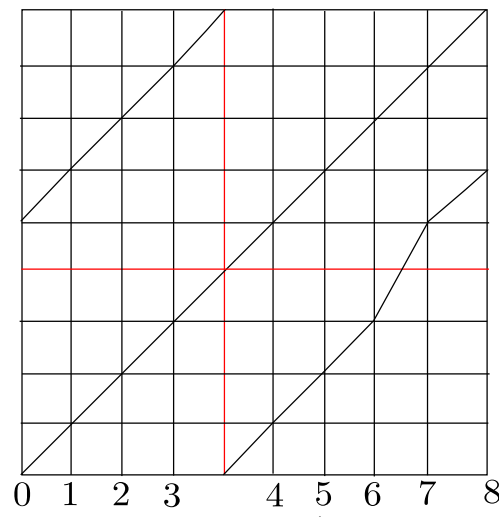

This map represents the dynamics of $\left.\sigma\right|_{\left[a_{-}, \underline{b} b_{+} \underline{a}\right]}$ with $a_{-} \underline{b}=0 \underline{1010101}$ and $b_{+} \underline{a}=1 \underline{1010110}$, where $a=\underline{0101011}$ and $\overline{\operatorname{Per}}(\vec{a})=7$.

Let us now consider $P^{5}$ :

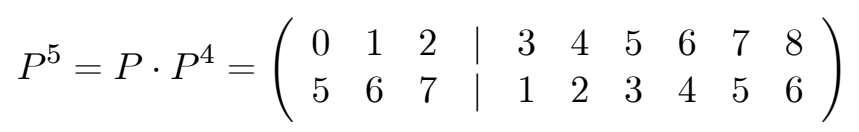

This permutation allows us to define a discontinuous map of the interval (like in the previous cases $p=3,5$ ) 


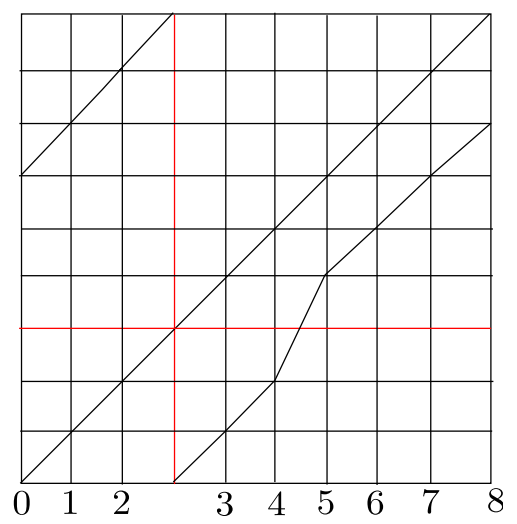

This map represents the dynamics of $\left.\sigma\right|_{\left[a_{-}, \underline{b}, b_{+} \underline{a}\right]}$ with $a_{-} \underline{b}=0 \underline{1101101}$ and $b_{+} \underline{a}=1 \underline{1101110}$, where $a=\underline{0110111}$ and $\operatorname{Per}(\bar{a})=7$.

Let us now consider $P^{6}$ :

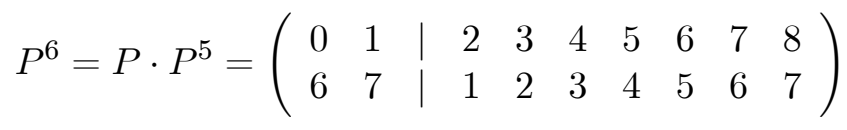

This permutation allows us to define a discontinuous map of the interval (like in the previous cases $p=3,5$ )

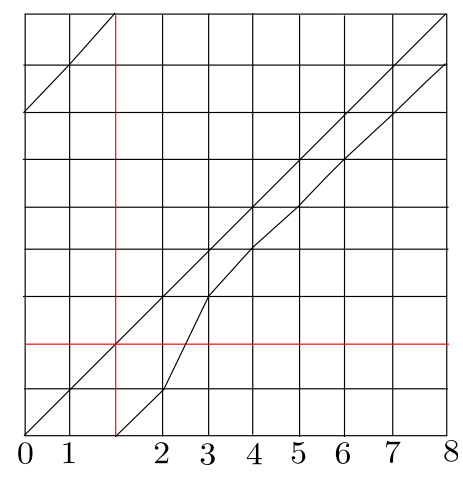

This map represents the dynamics of $\left.\sigma\right|_{\left[a_{-} \underline{b}, b_{+} \underline{a}\right]}$ with $a_{-} \underline{b}=0 \underline{1111101}$ and $b_{+} \underline{a}=11_{6} 0$,

where $a=\underline{01_{6}}$ and $\operatorname{Per}(a)=7$.

Let us now consider $P^{7}$ :

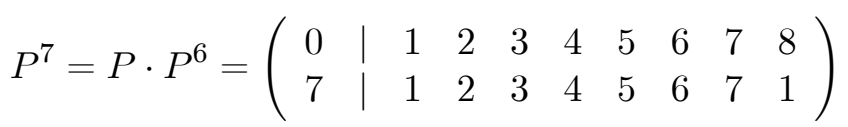

Using this permutation we cannot construct a dynamics that represents some subshift of the lexicographical world. 
Now, these six sequences are minimal periodic sequences with period 7 in $A_{\infty}^{\infty}$. These are: $\underline{0_{6} 1}, \underline{0001001}, \underline{0010101}, \underline{0101011}, \underline{0110111}$ and $\underline{01_{6}}$.

We observe that the other minimal sequences of period seven are:

1. $\underline{0000111}=\underline{a_{-} d_{+}}$with $a=\underline{0001}, d=\underline{110}$

2. $\underline{0001111}=\underline{a_{-} d_{+}}$with $a=\underline{001}, d=\underline{1110}$

3. $\underline{0000101}=\underline{a_{-} d_{+}}$with $a=\underline{0001}, d=\underline{100}$

4. $\underline{0011101}=\underline{a_{-} d_{+} a}$ with $a=\underline{01}, d=\underline{110}$

5. $\underline{0001011}=\underline{a_{-} d d_{+}}$with $a=\underline{001}, d=\underline{10}$

6. $\underline{0001101}=\underline{a_{-} d_{+}}$with $a=\underline{001}, d=\underline{1100}$

7. $\underline{0010111}=\underline{a_{-} d_{+}}$with $a=\underline{0011}, d=\underline{110}$

8. $\underline{0011111}=\underline{a_{-} d_{+}}$with $a=\underline{01}, d=\underline{11110}$

9. $\underline{0011011}=\underline{a_{-} d_{+}}$with $a=\underline{01}, d=\underline{11010}$

10. $\underline{0000011}=\underline{a_{-} d_{+}}$with $a=\underline{00001}, d=\underline{10}$

11. $\underline{0010011}=\underline{a_{-} d_{+}}$with $a=\underline{00101}, d=\underline{10}$

12. $\underline{0101111}=\underline{a_{-} d_{+}}$with $a=\underline{011}, d=\underline{1110}$

Accordingly with theorem A in [4] all of these sequences satisfies $h_{t o p}(\alpha, b(\alpha))=h_{t o p}(\underline{a}, \underline{d})$ and, from observation 2.0.10 at [4] we have: $h_{\text {top }}(\underline{a}, \underline{d})>0$.

We also observe that for any $\alpha \in \mathcal{A}_{\infty}^{\infty}$ we have $h_{\text {top }}(\alpha, b(\alpha))=0$.

Now, let us consider a sequence $a \in \mathcal{A}_{\infty}^{\infty}$ such that $\operatorname{per}(a)=p$. Accordingly with the proof of Theorem $\mathrm{C}$ (in [4]) for any $a \in \mathcal{A}_{\infty}^{\infty}$ as before, we can construct a discontinuous map $f_{a}: I \backslash\left\{c_{a}\right\} \rightarrow I, I=[0,1]$ (like the previous f) such that $I_{f_{a}}(0)=a_{-} \underline{b(a)}$ and $I_{f_{a}}(1)=(b(a))_{+}$. For this map, $f_{a}$, we have a decomposition of the interval $I=\bigcup_{i=1}^{p+2} I_{i}$, such that the associated graph to the dynamics of $f_{a}$ under this decomposition of the interval, $G\left(f_{a}\right)$, is equal to the graph associated to the previous $f\left(=G(f)\right.$ associated to $\left.0_{p-1} 1\right)$. This, in particular, implies that

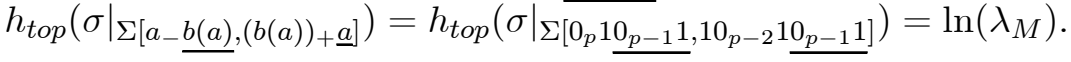


Observation 2.6. The result is also true for $p=2$. In this case $a=\underline{01} \in$ $\mathcal{A}_{\infty}^{\infty}$.

In fact, for $a=\underline{01}, b=b(a) \underline{10}$, we have $a_{-} \underline{b}=00 \underline{10}=0 \underline{01}$ and $b_{+} \underline{a}=1 \underline{10}$, so $0=a_{-} \underline{b}=0 \underline{01} \rightarrow \sigma(\underline{001})=\underline{01}=1 \rightarrow \sigma(\underline{01})=\underline{10}=2 \rightarrow \sigma(\underline{10})=\underline{01}=1 ;$ $b_{+} \underline{a}=1 \underline{10}=3 \rightarrow \sigma(1 \underline{10})=\underline{10}=2$.

Then we can define a discontinuous map of the interval

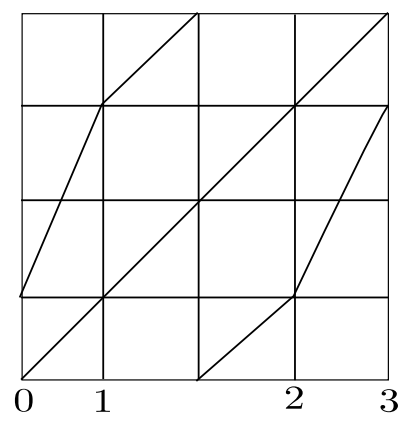

The characteristic polynomial associated to the respective graph is $p(\lambda)=$ $\lambda^{4}-2 \lambda^{2}$ then $p(\lambda)=0$ for $\lambda=0$ or $\lambda=\sqrt{2}$, hence $h_{\text {top }}\left(\left.\sigma\right|_{\Sigma\left[a_{-}, b_{+} \underline{a}\right]}\right)=$ $\log (\sqrt{2})=\frac{1}{2} \log (2)$

\section{The Algorithm}

In this section the algorithm that computes the entropy is described. The figure 3 shows the instructions of the algorithm. The first step consist in to generate the permutations $p^{0}, p^{1}, \ldots, p^{n-1}$, each permutation has $n+2$ elements indexed between $0 \ldots n+1$. The generation of $p^{0}, p^{1}, \ldots, p^{n-1}$ is performed by the instructions between lines 1 and 11 .

In the second step (line 12), the algorithm generates the graph associated to the permutation $p^{1}$ and then computes the maximum eigenvalue associated to it (line 13). The algorithm returns its natural logarithm (line $15)$. 


\section{Algorithm Entropy}

Input: $n \in \mathcal{N}, n$ a prime number

Output: $e \in \mathcal{R}, e$ entropy value if exists

-1 if not exists such entropy value

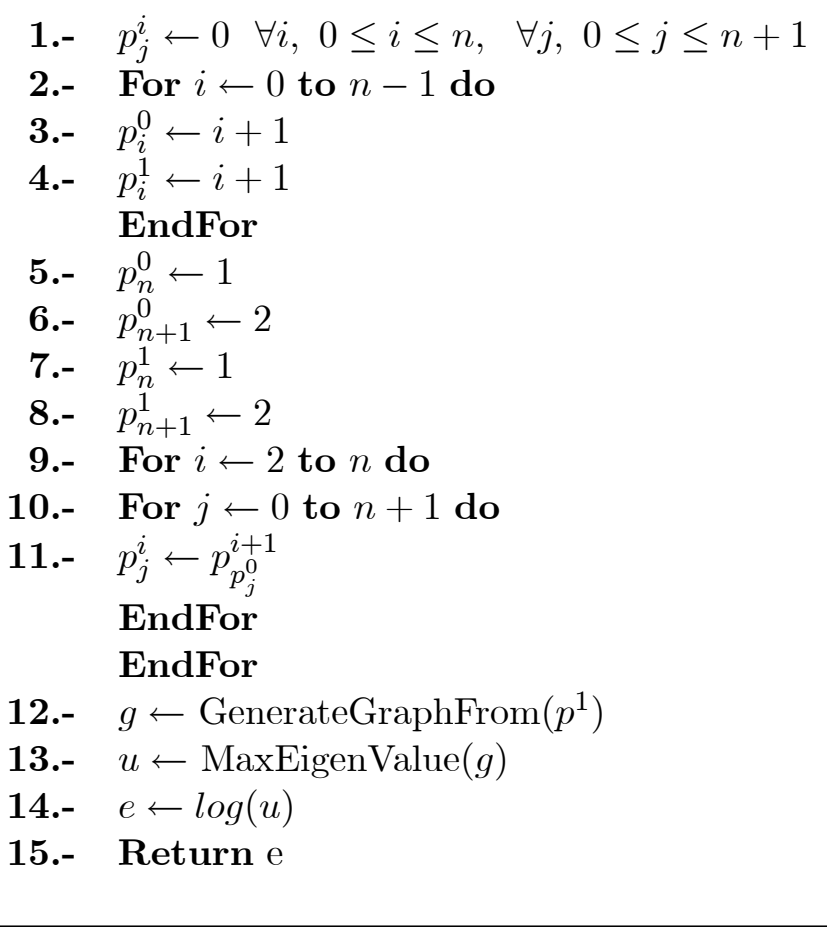

Figure : The Algorithm. 


\subsection{Cost of the Algorithm}

We analyze the efficiency of the algorithm in terms of the memory space and the number of operations. Both parameters are related with the value of $n$. In terms of space it is necessary to store the permutations, the graphs and the auxiliary vectors to compute the maximum eigenvalue.

\begin{tabular}{|l|r|c|}
\hline \multicolumn{1}{|c|}{ Steps } & \multicolumn{1}{|c|}{ Required Space } & Number of Operations \\
\hline Permutations & $(n+1) \times(n+2)$ & $\mathcal{O}\left(n^{2}\right)$ \\
\hline Graphs & $(n-1) \times((n+2) \times(n+2))$ & $\mathcal{O}\left(n^{2}\right)$ \\
\hline Eigenvalues & $(n-1) \times n \times(n \times n)$ & $\mathcal{O}\left(n^{3}\right)$ \\
\hline
\end{tabular}

Figure 3.1: Cost of the Algorithm

Figure 4 shows the required space and the number of operations of the different sections of the algorithm. The maximal number of operations correspond to compute the maximum eigenvalue that uses the method QR [10] (line 13) with a $\operatorname{cost} \mathcal{O}\left(n^{3}\right)$.

\section{Experimental Results}

The algorithm was implemented in C language under Linux OS. The computation of the maximum eigenvalues was performed with the GNU Scientific Library (GSL) [11]. The hardware we used is a Intel $3.60 \mathrm{GHz}$ with 4 Gib of RAM. Because the GSL function that computes the maximum eigenvalue of a matrix require 8 bytes (double) per element. The maximum value of $n$ is 20,123 because the space needed is $20,123 \times 20,123 \times 8=$ $3,239,481,032$ bytes. In the appendix A are reported the entropy values for prime numbers from $p=3$ and up to $p=9973$. 


\section{Discussion}

The main motivation for our studies, concerning the standard quadratic family of Lorenz maps, is to prove the isentrope conjecture for this family. This conjecture were established by John Milnor ([28]) in a different context. Essentially it consists in to prove that the set $H_{0}=\left\{(\mu, \nu) ; h_{t o p}(\mu, \nu)=\right.$ $0\}$ is a connected set.

The main result, that we have verify here, allows us to advance a step further in to find the boundary of the set $H_{0}$ in the region, $B$, bounded by the curves $\{(0, \nu) ; 0 \leq \nu \leq 1\},\left\{(\mu, \nu) ; \nu=\frac{1+\sqrt{1+4 \mu}}{2}, 0 \leq \mu \leq 2\right\}$, $\{(\mu, 0) ; 0 \leq \mu \leq 1\},\left\{(\mu, \nu) ; \mu=\frac{1+\sqrt{1+4 \nu}}{2}, 0 \leq \nu \leq 2\right\}$. We note that for any $(\mu, \nu) \in\left\{(\mu, \nu) ; \nu=\frac{1+\sqrt{1+4 \mu}}{2}, 0 \leq \mu \leq 2\right\}$, we have $b(\mu, \nu)=\underline{1}$ and for any $(\mu, \nu) \in\left\{(\mu, \nu) ; \mu=\frac{1+\sqrt{1+4 \nu}}{2}, 0 \leq \nu \leq 2\right\}$ we have $a(\mu, \nu)=\underline{0}$.

In fact, accordingly with [5] we have the following results in the quadratic family.

Let us consider $a \in \mathcal{A}_{\infty}^{\infty}$ and $b=b(a)=\sup \left\{\sigma^{j}(a) ; j \in N\right\}$.

Let $B_{1}\left(\underline{0}, a_{-} \underline{b}, b_{+} \underline{a}, \underline{1}\right)=\left\{(\mu, \nu) \in B ; \underline{0} \leq I(-\mu) \leq a_{-} \underline{b}, b_{+} \underline{a} \leq I(\nu) \leq\right.$ $\underline{1}\}, B_{2}\left(a_{-} \underline{b}, \underline{a}, b_{+} \underline{a}, \underline{1}\right)=\left\{(\mu, \nu) \in B ; a_{-} \underline{b} \leq I(-\mu) \leq \underline{a}, b_{+} \underline{a}<I(\nu) \leq \underline{1}\right\}$, $B_{3}\left(\underline{0}, a_{-} \underline{b}, \underline{b}, b_{+} \underline{a}\right)=\left\{(\mu, \nu) \in B ; \underline{0} \leq I(-\mu)<a_{-} \underline{b}, \underline{b} \leq I(\nu) \leq b_{+} \underline{a}\right\}$. For any $(\mu, \nu) \in B_{1} \cup B_{2} \cup B_{3}$ let $\Lambda(\mu, \nu)=\bigcap_{j=0}^{\infty} F_{\mu, \nu}^{-j}([-\mu, \nu])$

Proposition 5.1. For $(\mu, \nu) \in B_{1} \cup B_{2} \cup B_{3}$ we have that $h_{\text {top }}\left(\left.F_{\mu, \nu}\right|_{\Lambda(\mu, \nu)}\right)>0$

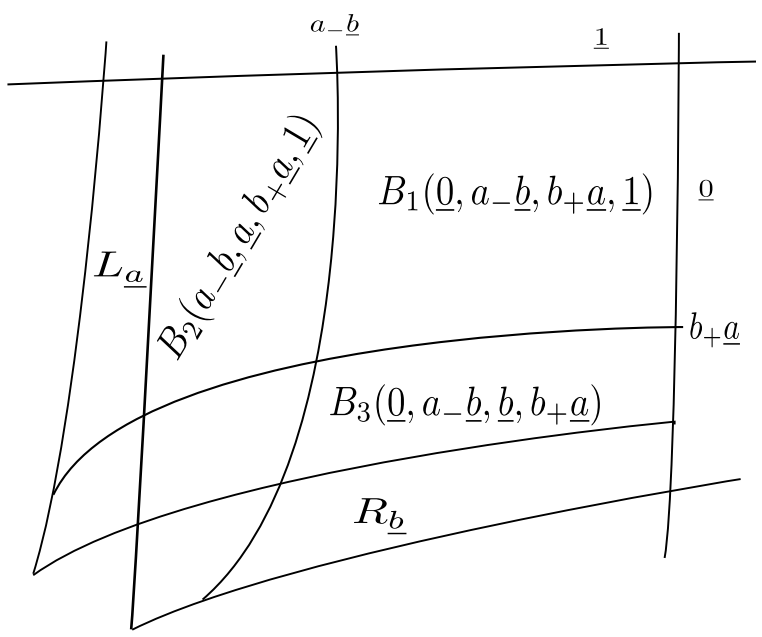

Figure 5: Regions $B_{1} \cup B_{2} \cup B_{3}$ 
A computer verification for the value of the Topological Entropy... 461

Observation 5.2. 1 . The set $B_{2}\left(a_{-} \underline{b}, \underline{a}, b_{+} \underline{a}, \underline{1}\right)$ include the region $L_{\underline{a}}=$ $\left\{(\mu, \nu) ; I(-\mu)=\underline{a}, b_{+} \underline{a}<I(\nu) \leq \underline{1}\right\}$ which has a non-empty interior (see $[20])$.

2. The set $B_{3}\left(\underline{0}, a_{-} \underline{b}, \underline{b}, b_{+} \underline{a}\right)$ include the region $R_{\underline{b}}=\{(\mu, \nu) ; I(\nu)=$ $\left.\underline{b}, \underline{0} \leq I(-\mu)<a_{-} \underline{b}\right\}$ which has a non-empty interior.

3. The explanation of the figures in this section were given at [20] and [31]

For $a \in A_{\infty}^{\infty}$ let $C_{1}\left(\underline{a}, b_{+} \underline{a}\right)=\left\{(\mu, \nu) \in B ; I(-\mu)=\underline{a}, I(\nu)=b_{+} \underline{a}\right\}$, $C_{2}(\underline{a}, \underline{b})=\{(\mu, \nu) \in B ; I(-\mu)=\underline{a}, I(\nu)=\underline{b}\}, C_{3}\left(a_{-} \underline{b}, \underline{b}\right)=\{(\mu, \nu) \in$ $\left.B ; I(-\mu)=a_{-} \underline{b}, I(\nu)=\underline{b}\right\}$

Proposition 5.3. For any $(\mu, \nu) \in C_{1} \cup C_{2} \cup C_{3}$, we have that $h_{\text {top }}\left(\left.F_{\mu, \nu}\right|_{\Lambda(\mu, \nu)}\right)=0$

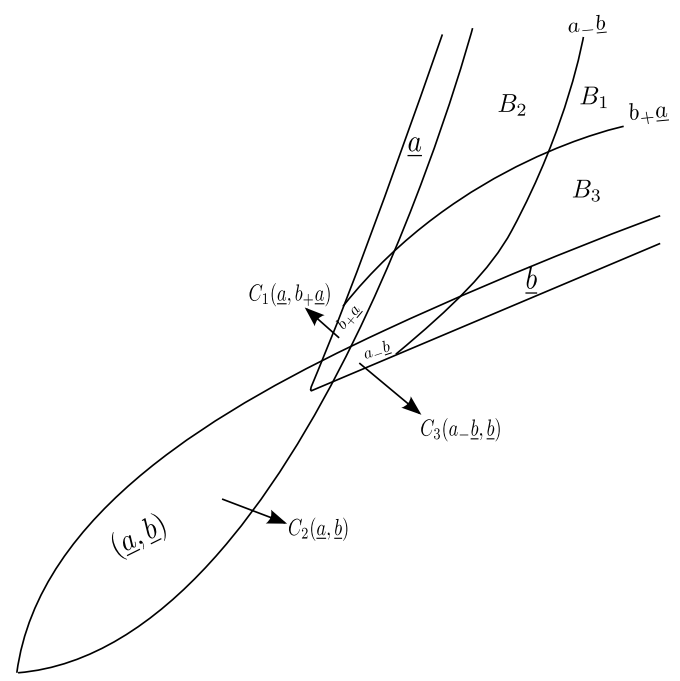

Figure 6: Region $C_{1} \cup C_{2} \cup C_{3}$

Now, let us denote by $B(a, b)$, for $a \in \mathcal{A}_{\infty}^{\infty}$ and $b=b(a)$ the region bounded by the curves $\gamma_{a, b_{+} \underline{a}}=\left\{(\mu, \nu) ; a(\mu, \nu)=a ; b(\mu, \nu)=b_{+} \underline{a}\right\}, \gamma_{a_{-} \underline{b}, \underline{b}}=$ $\left\{(\mu, \nu) ; a(\mu, \nu)=a_{-} \underline{b} ; b(\mu, \nu)=\underline{b}\right\}, \gamma_{b_{+} \underline{a}}=\left\{(\mu, \nu) ; a_{-} \underline{b} \leq a(\mu, \nu) \leq \underline{a}\right\}$, and $\gamma_{a_{-} \underline{b}}=\left\{(\mu, \nu) ; \underline{b} \leq b(\mu, \nu) \leq b_{+} \underline{a}\right\}$. 


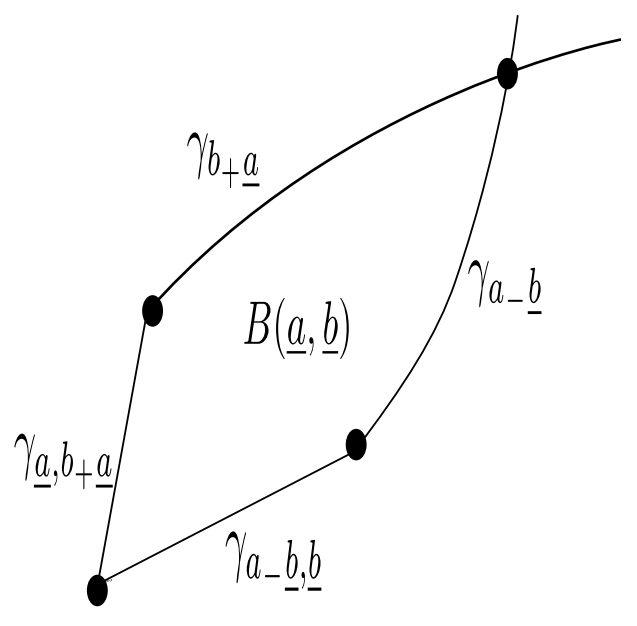

Figure 7: Region $B(a, b)$

The maximal value for the topological entropy in the region $B(a, b)$ is obtained at the intersection of the curves $\gamma_{b_{+} \underline{a}}$ and $\gamma_{a_{-} \underline{b}}$ and this value is exactly $h_{\text {top }}\left(a_{-} \underline{b}, b_{+} \underline{a}\right)=\frac{1}{\operatorname{Per}(a)} \ln (2)$. That is, our values are an upper bound for the values for the topological entropy $h_{t o p}(\mu, \nu)$ for $(\mu, \nu) \in$ $B(a, b)$.

Now, let us consider the doubling period sequences $\underline{a}_{1}=a_{-} b_{+}$and $\underline{b_{1}}=\underline{b_{+} a_{-}}=\underline{b\left(a_{1}\right)}$. Let us define the corresponding regions $\underline{C_{1}}\left(\underline{a_{1}}, \overline{\left(b_{1}\right)}+\underline{a_{1}}\right)$, $\overline{C_{2}}\left(\underline{a_{1}}, \underline{\left.b_{1}\right)}, C_{3} \overline{\left(\left(a_{1}\right)\right.}-\underline{b_{1}}, \underline{b_{1}}\right), B_{1}\left(\underline{0},\left(a_{1}\right)_{-} \underline{b_{1}},\left(b_{1}\right)_{+} \underline{a_{1}}, \underline{1}\right), B_{2}\left(\left(a_{1}\right)-\underline{b_{1}}, \underline{a_{1}},\left(b_{1}\right)_{+} \underline{a_{1}}, \underline{1}\right)$, $B_{3}\left(\underline{0},\left(a_{1}\right)-\underline{b_{1}}, \underline{b_{1}},\left(b_{1}\right)+\underline{a_{1}}\right)$ and $B\left(\underline{a_{1}}, \underline{\left.b_{1}\right)}\right.$. All of these regions are included in $B(\underline{a}, \underline{b})$ (see figure 8$)$

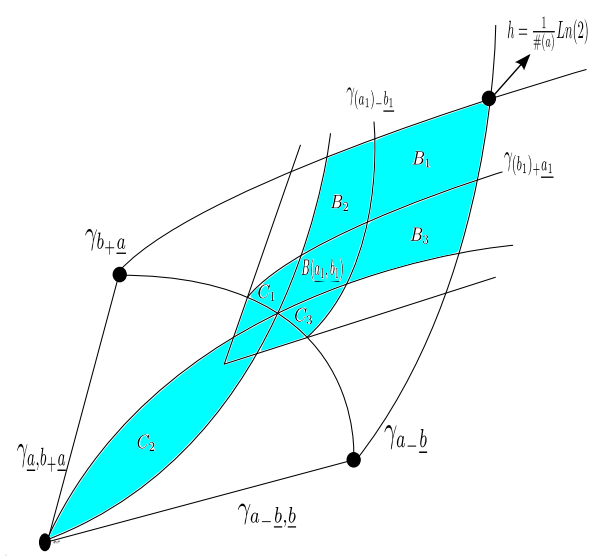

Figure 8: Region $B\left(a_{1}, b_{1}\right)$

In this case for the parameter value $(\bar{\mu}, \bar{\nu})=\gamma_{a_{-} \underline{b}} \cap \gamma_{b_{+} \underline{a}}$ and $\left(\overline{\mu_{1}}, \overline{\nu_{1}}\right)=$ $\gamma_{\left(a_{1}\right)_{-} \underline{b_{1}}} \cap \gamma_{\left(b_{1}\right)_{+} \underline{a_{1}}}$ we have that $h_{t o p}\left(\overline{\mu_{1}}, \overline{\nu_{1}}\right)=\frac{1}{2 \#(a)} \operatorname{Ln}(2)=\frac{1}{2} h_{t o p}(\bar{\mu}, \bar{\nu})$. 
That is for any element $(\mu, \nu) \in B\left(\underline{a_{1}}, \underline{b_{1}}\right)$ we have $h_{t o p}(\mu, \nu) \leq \frac{1}{2} h_{t o p}(\bar{\mu}, \bar{\nu})$. Moreover, for $(\mu, \nu) \in B_{1}\left(\underline{0},\left(a_{1}\right)_{-} \underline{b_{1}},\left(b_{1}\right)_{+} \underline{a_{1}}, \underline{1}\right) \cup B_{2}\left(\left(a_{1}\right)_{-} \underline{b_{1}}, \underline{a_{1}},\left(b_{1}\right)_{+} \underline{a_{1}}, \underline{1}\right) \cup$ $B_{3}\left(\underline{0},\left(a_{1}\right)-\underline{b_{1}}, \underline{b_{1}},\left(b_{1}\right)+\underline{a_{1}}\right)$ we have $h_{\text {top }}(\mu, \nu)>0$.

Also for any $(\mu, \nu) \in \overline{C_{1}}\left(\underline{a_{1}},\left(b_{1}\right)+\underline{a_{1}}\right) \cup C_{2}\left(\underline{a_{1}}, \underline{b_{1}}\right) \cup C_{3}\left(\left(a_{1}\right)_{-} \underline{b_{1}}, \underline{b_{1}}\right)$ we have $h_{\text {top }}(\mu, \nu)=0$.

Therefore, we can connect any point of $C_{2}(\underline{a}, \underline{b})$ with any point of $C_{1}\left(\underline{a_{1}},\left(b_{1}\right)+\underline{a_{1}}\right) \cup C_{2}\left(\underline{a_{1}}, \underline{b_{1}}\right) \cup C_{3}\left(\left(a_{1}\right)-\underline{b_{1}}, \underline{b_{1}}\right)$ with a path completely contained in the region $\left\{(\mu, \nu) ; h_{\text {top }}(\mu, \nu)=0\right\}$.

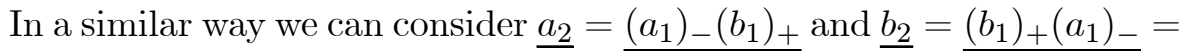
$b\left(a_{2}\right)$ and to proceed to construct regions $C_{1}, C_{2}, C_{3}, B_{1}, B_{2}, \overline{B_{3}, B \text { associ- }}$ ated to $\left(\underline{a_{2}}, \underline{b_{2}}\right)$ and so on. We observe that for elements $(\mu, \nu) \in B\left(\underline{a_{2}}, \underline{b_{2}}\right)$ we will have $h_{t o p}(\mu, \nu) \leq \frac{1}{2 \#\left(a_{1}\right)} \operatorname{Ln}(2)=\frac{1}{2^{2} \#(a)} \operatorname{Ln}(2)$; that is we have a Lower bound for the topological entropy than in $B\left(\underline{a_{1}}, \underline{b_{1}}\right)$.

Successively, for $a_{n+1}=\left(a_{n}\right)_{-}\left(b_{n}\right)_{+}, b_{n+1}=\left(b_{n}\right)_{+}\left(a_{n}\right)_{-}=b\left(a_{n+1}\right)$ we construct similar regions $C_{1}, \overline{C_{2}, C_{3}, B_{1}}, \overline{B_{2}, B}{ }_{3}, \overline{B \text {. }}$

Moreover, any element in

$C_{1}\left(\underline{a_{n+1}},\left(b_{n+1}\right)+\underline{a_{n+1}}\right) \cup C_{2}\left(\underline{a_{n+1}}, \underline{b_{n+1}}\right) \cup C_{3}\left(\left(a_{n+1}\right)-\underline{b_{n+1}}, \underline{b_{n+1}}\right)$ can be connected to any element of $C_{2} \overline{(\underline{a}, \underline{b})} \overline{\text { by an }}$ arc $\gamma$ completely contained in the region $\left\{(\mu, \nu) ; h_{\text {top }}(\mu, \nu)=0\right\}$. In this way we observe that there is a good chance to obtain a proof that the isentrope $\left\{(\mu, \nu) ; h_{t o p}(\mu, \nu)=0\right\}$ is a arc connected set.

\section{Materials and Methods}

The algorithm for the computation of the topological entropy is know, see for instance [6] or [16].

Acknowledgments: Part of this paper is an outgrowth of research of S. Aranzubia and R. Labarca during a visit to IMPA Brasil. Also, R. Labarca acknowledge the kind hospitality of the Imperial College while preparing part of this work and the finnantial support of project USA1656(CHILE). 


\section{A. Entropy Values for Prime Numbers Between 3 and 9923}

Entropy Values for Prime Numbers Between 3 and 751

\begin{tabular}{|c|c|c|c|c|c|c|c|}
\hline Value & Entropy & Value & Entropy & Value & Entropy & Value & Entropy \\
\hline 3 & 0.231048000 & 5 & 0.138631000 & 7 & 0.099021500 & 11 & 0.063012400 \\
\hline 13 & 0.053322700 & 17 & 0.040777200 & 19 & 0.036486200 & 23 & 0.030141200 \\
\hline 29 & 0.023902100 & 31 & 0.022358200 & 37 & 0.018733400 & 41 & 0.016906300 \\
\hline 43 & 0.016119400 & 47 & 0.014750700 & 53 & 0.013074200 & 59 & 0.011750700 \\
\hline 61 & 0.011365200 & 67 & 0.010346300 & 71 & 0.009762190 & 73 & 0.009494780 \\
\hline 79 & 0.008771420 & 83 & 0.008355000 & 89 & 0.007789580 & 97 & 0.007144420 \\
\hline 101 & 0.006866370 & 103 & 0.006727320 & 107 & 0.006478970 & 109 & 0.006359730 \\
\hline 113 & 0.006131170 & 127 & 0.005455090 & 131 & 0.005295950 & 137 & 0.005057190 \\
\hline 139 & 0.004987540 & 149 & 0.004649180 & 151 & 0.004589450 & 157 & 0.004410260 \\
\hline 163 & 0.004250950 & 167 & 0.004151370 & 173 & 0.004001980 & 179 & 0.003872490 \\
\hline 181 & 0.003832650 & 191 & 0.003633390 & 193 & 0.003593540 & 197 & 0.003513820 \\
\hline 199 & 0.003483920 & 211 & 0.003284600 & 223 & 0.003105170 & 227 & 0.003055330 \\
\hline 229 & 0.003025420 & 233 & 0.002975570 & 239 & 0.002895800 & 241 & 0.002875860 \\
\hline 251 & 0.002766170 & 257 & 0.002696360 & 263 & 0.002636520 & 269 & 0.002576680 \\
\hline 271 & 0.002556730 & 277 & 0.002506860 & 281 & 0.002466950 & 283 & 0.002447000 \\
\hline 293 & 0.002367200 & 307 & 0.002257450 & 311 & 0.002227520 & 313 & 0.002217540 \\
\hline 317 & 0.002187610 & 331 & 0.002097800 & 337 & 0.002057880 & 347 & 0.001998000 \\
\hline 349 & 0.001988020 & 353 & 0.001968060 & 359 & 0.001928140 & 367 & 0.001888220 \\
\hline 373 & 0.001858270 & 379 & 0.001828330 & 383 & 0.001808360 & 389 & 0.001778420 \\
\hline 397 & 0.001748470 & 401 & 0.001728510 & 409 & 0.001698560 & 419 & 0.001658620 \\
\hline 421 & 0.001648640 & 431 & 0.001608710 & 433 & 0.001598720 & 439 & 0.001578750 \\
\hline 443 & 0.001568770 & 449 & 0.001538820 & 457 & 0.001518850 & 461 & 0.001498880 \\
\hline 463 & 0.001498880 & 467 & 0.001488890 & 479 & 0.001448950 & 487 & 0.001418990 \\
\hline 491 & 0.001409010 & 499 & 0.001389030 & 503 & 0.001379050 & 509 & 0.001359080 \\
\hline 521 & 0.001329120 & 523 & 0.001329120 & 541 & 0.001279180 & 547 & 0.001269190 \\
\hline 557 & 0.001249220 & 563 & 0.001229240 & 569 & 0.001219260 & 571 & 0.001209270 \\
\hline 577 & 0.001199280 & 587 & 0.001179300 & 593 & 0.001169320 & 599 & 0.001159330 \\
\hline 601 & 0.001149340 & 607 & 0.001139350 & 613 & 0.001129360 & 617 & 0.001119370 \\
\hline 619 & 0.001119370 & 631 & 0.001099400 & 641 & 0.001079420 & 643 & 0.001079420 \\
\hline 647 & 0.001069430 & 653 & 0.001059440 & 659 & 0.001049450 & 661 & 0.001049450 \\
\hline 673 & 0.001029470 & 677 & 0.001019480 & 683 & 0.001019480 & 691 & 0.000999500 \\
\hline 701 & 0.000989510 & 709 & 0.000979520 & 719 & 0.000959539 & 727 & 0.000949549 \\
\hline 733 & 0.000949549 & 739 & 0.000939558 & 743 & 0.000929568 & 751 & 0.000919577 \\
\hline
\end{tabular}


A computer verification for the value of the Topological Entropy... 465

Entropy Values for Prime Numbers Between 757 and 1697

\begin{tabular}{|c|c|c|c|c|c|c|c|}
\hline Value & Entropy & Value & Entropy & Value & Entropy & Value & Entropy \\
\hline 757 & 0.000919577 & 761 & 0.000909586 & 769 & 0.000899595 & 773 & 0.000899595 \\
\hline 787 & 0.000879613 & 797 & 0.000869622 & 809 & 0.000859630 & 811 & 0.000859630 \\
\hline 821 & 0.000839647 & 823 & 0.000839647 & 827 & 0.000839647 & 829 & 0.000839647 \\
\hline 839 & 0.000829656 & 853 & 0.000809672 & 857 & 0.000809672 & 859 & 0.000809672 \\
\hline 863 & 0.000799680 & 877 & 0.000789688 & 881 & 0.000789688 & 883 & 0.000789688 \\
\hline 887 & 0.000779696 & 907 & 0.000759711 & 911 & 0.000759711 & 919 & 0.000749719 \\
\hline 929 & 0.000749719 & 937 & 0.000739726 & 941 & 0.000739726 & 947 & 0.000729734 \\
\hline 953 & 0.000729734 & 967 & 0.000719741 & 971 & 0.000709748 & 977 & 0.000709748 \\
\hline 983 & 0.000709748 & 991 & 0.000699755 & 997 & 0.000699755 & 1009 & 0.000689762 \\
\hline 1013 & 0.000679769 & 1019 & 0.000679769 & 1021 & 0.000679769 & 1031 & 0.000669776 \\
\hline 1033 & 0.000669776 & 1039 & 0.000669776 & 1049 & 0.000659782 & 1051 & 0.000659782 \\
\hline 1061 & 0.000649789 & 1063 & 0.000649789 & 1069 & 0.000649789 & 1087 & 0.000639795 \\
\hline 1091 & 0.000639795 & 1093 & 0.000629802 & 1097 & 0.000629802 & 1103 & 0.000629802 \\
\hline 1109 & 0.000629802 & 1117 & 0.000619808 & 1123 & 0.000619808 & 1129 & 0.000609814 \\
\hline 1151 & 0.000599820 & 1153 & 0.000599820 & 1163 & 0.000599820 & 1171 & 0.000589826 \\
\hline 1181 & 0.000589826 & 1187 & 0.000579832 & 1193 & 0.000579832 & 1201 & 0.000579832 \\
\hline 1213 & 0.000569838 & 1217 & 0.000569838 & 1223 & 0.000569838 & 1229 & 0.000559843 \\
\hline 1231 & 0.000559843 & 1237 & 0.000559843 & 1249 & 0.000559843 & 1259 & 0.000549849 \\
\hline 1277 & 0.000539854 & 1279 & 0.000539854 & 1283 & 0.000539854 & 1289 & 0.000539854 \\
\hline 1291 & 0.000539854 & 1297 & 0.000529860 & 1301 & 0.000529860 & 1303 & 0.000529860 \\
\hline 1307 & 0.000529860 & 1319 & 0.000529860 & 1321 & 0.000519865 & 1327 & 0.000519865 \\
\hline 1361 & 0.000509870 & 1367 & 0.000509870 & 1373 & 0.000499875 & 1381 & 0.000499875 \\
\hline 1399 & 0.000499875 & 1409 & 0.000489880 & 1423 & 0.000489880 & 1427 & 0.000489880 \\
\hline 1429 & 0.000489880 & 1433 & 0.000479885 & 1439 & 0.000479885 & 1447 & 0.000479885 \\
\hline 1451 & 0.000479885 & 1453 & 0.000479885 & 1459 & 0.000479885 & 1471 & 0.000469890 \\
\hline 1481 & 0.000469890 & 1483 & 0.000469890 & 1487 & 0.000469890 & 1489 & 0.000469890 \\
\hline 1493 & 0.000459894 & 1499 & 0.000459894 & 1511 & 0.000459894 & 1523 & 0.000459894 \\
\hline 1531 & 0.000449899 & 1543 & 0.000449899 & 1549 & 0.000449899 & 1553 & 0.000449899 \\
\hline 1559 & 0.000439903 & 1567 & 0.000439903 & 1571 & 0.000439903 & 1579 & 0.000439903 \\
\hline 1583 & 0.000439903 & 1597 & 0.000429908 & 1601 & 0.000429908 & 1607 & 0.000429908 \\
\hline 1609 & 0.000429908 & 1613 & 0.000429908 & 1619 & 0.000429908 & 1621 & 0.000429908 \\
\hline 1627 & 0.000429908 & 1637 & 0.000419912 & 1657 & 0.000419912 & 1663 & 0.000419912 \\
\hline 1667 & 0.000419912 & 1669 & 0.000419912 & 1693 & 0.000409916 & 1697 & 0.000409916 \\
\hline
\end{tabular}


Entropy Values for Prime Numbers Between 1699 and 2719

\begin{tabular}{|c|c|c|c|c|c|c|c|}
\hline Value & Entropy & Value & Entropy & Value & Entropy & Value & Entropy \\
\hline 1699 & 0.000409916 & 1709 & 0.000409916 & 1721 & 0.000399920 & 1723 & 0.000399920 \\
\hline 1733 & 0.000399920 & 1741 & 0.000399920 & 1747 & 0.000399920 & 1753 & 0.000399920 \\
\hline 1759 & 0.000389924 & 1777 & 0.000389924 & 1783 & 0.000389924 & 1787 & 0.000389924 \\
\hline 1789 & 0.000389924 & 1801 & 0.000379928 & 1811 & 0.000379928 & 1823 & 0.000379928 \\
\hline 1831 & 0.000379928 & 1847 & 0.000379928 & 1861 & 0.000369932 & 1867 & 0.000369932 \\
\hline 1871 & 0.000369932 & 1873 & 0.000369932 & 1877 & 0.000369932 & 1879 & 0.000369932 \\
\hline 1889 & 0.000369932 & 1901 & 0.000359935 & 1907 & 0.000359935 & 1913 & 0.000359935 \\
\hline 1931 & 0.000359935 & 1933 & 0.000359935 & 1949 & 0.000359935 & 1951 & 0.000359935 \\
\hline 1973 & 0.000349939 & 1979 & 0.000349939 & 1987 & 0.000349939 & 1993 & 0.000349939 \\
\hline 1997 & 0.000349939 & 1999 & 0.000349939 & 2003 & 0.000349939 & 2011 & 0.000339942 \\
\hline 2017 & 0.000339942 & 2027 & 0.000339942 & 2029 & 0.000339942 & 2039 & 0.000339942 \\
\hline 2053 & 0.000339942 & 2063 & 0.000339942 & 2069 & 0.000339942 & 2081 & 0.000329946 \\
\hline 2083 & 0.000329946 & 2087 & 0.000329946 & 2089 & 0.000329946 & 2099 & 0.000329946 \\
\hline 2111 & 0.000329946 & 2113 & 0.000329946 & 2129 & 0.000329946 & 2131 & 0.000329946 \\
\hline 2137 & 0.000319949 & 2141 & 0.000319949 & 2143 & 0.000319949 & 2153 & 0.000319949 \\
\hline 2161 & 0.000319949 & 2179 & 0.000319949 & 2203 & 0.000309952 & 2207 & 0.000309952 \\
\hline 2213 & 0.000309952 & 2221 & 0.000309952 & 2237 & 0.000309952 & 2239 & 0.000309952 \\
\hline 2243 & 0.000309952 & 2251 & 0.000309952 & 2267 & 0.000309952 & 2269 & 0.000309952 \\
\hline 2273 & 0.000299955 & 2281 & 0.000299955 & 2287 & 0.000299955 & 2293 & 0.000299955 \\
\hline 2297 & 0.000299955 & 2309 & 0.000299955 & 2311 & 0.000299955 & 2333 & 0.000299955 \\
\hline 2339 & 0.000299955 & 2341 & 0.000299955 & 2347 & 0.000299955 & 2351 & 0.000289958 \\
\hline 2357 & 0.000289958 & 2371 & 0.000289958 & 2377 & 0.000289958 & 2381 & 0.000289958 \\
\hline 2383 & 0.000289958 & 2389 & 0.000289958 & 2393 & 0.000289958 & 2399 & 0.000289958 \\
\hline 2411 & 0.000289958 & 2417 & 0.000289958 & 2423 & 0.000289958 & 2437 & 0.000279961 \\
\hline 2441 & 0.000279961 & 2447 & 0.000279961 & 2459 & 0.000279961 & 2467 & 0.000279961 \\
\hline 2473 & 0.000279961 & 2477 & 0.000279961 & 2503 & 0.000279961 & 2521 & 0.000269964 \\
\hline 2531 & 0.000269964 & 2539 & 0.000269964 & 2543 & 0.000269964 & 2549 & 0.000269964 \\
\hline 2551 & 0.000269964 & 2557 & 0.000269964 & 2579 & 0.000269964 & 2591 & 0.000269964 \\
\hline 2593 & 0.000269964 & 2609 & 0.000269964 & 2617 & 0.000259966 & 2621 & 0.000259966 \\
\hline 2633 & 0.000259966 & 2647 & 0.000259966 & 2657 & 0.000259966 & 2659 & 0.000259966 \\
\hline 2663 & 0.000259966 & 2671 & 0.000259966 & 2677 & 0.000259966 & 2683 & 0.000259966 \\
\hline 2687 & 0.000259966 & 2689 & 0.000259966 & 2693 & 0.000259966 & 2699 & 0.000259966 \\
\hline 2707 & 0.000259966 & 2711 & 0.000259966 & 2713 & 0.000259966 & 2719 & 0.000249969 \\
\hline
\end{tabular}


A computer verification for the value of the Topological Entropy... 467

Entropy Values for Prime Numbers Between 2729 and 3803

\begin{tabular}{|c|c|c|c|c|c|c|c|}
\hline Value & Entropy & Value & Entropy & Value & Entropy & Value & Entropy \\
\hline 2729 & 0.000249969 & 2731 & 0.000249969 & 2741 & 0.000249969 & 2749 & 0.000249969 \\
\hline 2753 & 0.000249969 & 2767 & 0.000249969 & 2777 & 0.000249969 & 2789 & 0.000249969 \\
\hline 2791 & 0.000249969 & 2797 & 0.000249969 & 2801 & 0.000249969 & 2803 & 0.000249969 \\
\hline 2819 & 0.000249969 & 2833 & 0.000239971 & 2837 & 0.000239971 & 2843 & 0.000239971 \\
\hline 2851 & 0.000239971 & 2857 & 0.000239971 & 2861 & 0.000239971 & 2879 & 0.000239971 \\
\hline 2887 & 0.000239971 & 2897 & 0.000239971 & 2903 & 0.000239971 & 2909 & 0.000239971 \\
\hline 2917 & 0.000239971 & 2927 & 0.000239971 & 2939 & 0.000239971 & 2953 & 0.000229974 \\
\hline 2957 & 0.000229974 & 2963 & 0.000229974 & 2969 & 0.000229974 & 2971 & 0.000229974 \\
\hline 2999 & 0.000229974 & 3001 & 0.000229974 & 3011 & 0.000229974 & 3019 & 0.000229974 \\
\hline 3023 & 0.000229974 & 3037 & 0.000229974 & 3041 & 0.000229974 & 3049 & 0.000229974 \\
\hline 3061 & 0.000229974 & 3067 & 0.000229974 & 3079 & 0.000229974 & 3083 & 0.000219976 \\
\hline 3089 & 0.000219976 & 3109 & 0.000219976 & 3119 & 0.000219976 & 3121 & 0.000219976 \\
\hline 3137 & 0.000219976 & 3163 & 0.000219976 & 3167 & 0.000219976 & 3169 & 0.000219976 \\
\hline 3181 & 0.000219976 & 3187 & 0.000219976 & 3191 & 0.000219976 & 3203 & 0.000219976 \\
\hline 3209 & 0.000219976 & 3217 & 0.000219976 & 3221 & 0.000219976 & 3229 & 0.000209978 \\
\hline 3251 & 0.000209978 & 3253 & 0.000209978 & 3257 & 0.000209978 & 3259 & 0.000209978 \\
\hline 3271 & 0.000209978 & 3299 & 0.000209978 & 3301 & 0.000209978 & 3307 & 0.000209978 \\
\hline 3313 & 0.000209978 & 3319 & 0.000209978 & 3323 & 0.000209978 & 3329 & 0.000209978 \\
\hline 3331 & 0.000209978 & 3343 & 0.000209978 & 3347 & 0.000209978 & 3359 & 0.000209978 \\
\hline 3361 & 0.000209978 & 3371 & 0.000209978 & 3373 & 0.000209978 & 3389 & 0.000199980 \\
\hline 3391 & 0.000199980 & 3407 & 0.000199980 & 3413 & 0.000199980 & 3433 & 0.000199980 \\
\hline 3449 & 0.000199980 & 3457 & 0.000199980 & 3461 & 0.000199980 & 3463 & 0.000199980 \\
\hline 3467 & 0.000199980 & 3469 & 0.000199980 & 3491 & 0.000199980 & 3499 & 0.000199980 \\
\hline 3511 & 0.000199980 & 3517 & 0.000199980 & 3527 & 0.000199980 & 3529 & 0.000199980 \\
\hline 3533 & 0.000199980 & 3539 & 0.000199980 & 3541 & 0.000199980 & 3547 & 0.000199980 \\
\hline 3557 & 0.000189982 & 3559 & 0.000189982 & 3571 & 0.000189982 & 3581 & 0.000189982 \\
\hline 3583 & 0.000189982 & 3593 & 0.000189982 & 3607 & 0.000189982 & 3613 & 0.000189982 \\
\hline 3617 & 0.000189982 & 3623 & 0.000189982 & 3631 & 0.000189982 & 3637 & 0.000189982 \\
\hline 3643 & 0.000189982 & 3659 & 0.000189982 & 3671 & 0.000189982 & 3673 & 0.000189982 \\
\hline 3677 & 0.000189982 & 3691 & 0.000189982 & 3697 & 0.000189982 & 3701 & 0.000189982 \\
\hline 3709 & 0.000189982 & 3719 & 0.000189982 & 3727 & 0.000189982 & 3733 & 0.000189982 \\
\hline 3739 & 0.000189982 & 3761 & 0.000179984 & 3767 & 0.000179984 & 3769 & 0.000179984 \\
\hline 3779 & 0.000179984 & 3793 & 0.000179984 & 3797 & 0.000179984 & 3803 & 0.000179984 \\
\hline
\end{tabular}


Entropy Values for Prime Numbers Between 3821 and 4943

\begin{tabular}{|c|c|c|c|c|c|c|c|}
\hline Value & Entropy & Value & Entropy & Value & Entropy & Value & Entropy \\
\hline 3821 & 0.000179984 & 3823 & 0.000179984 & 3833 & 0.000179984 & 3847 & 0.000179984 \\
\hline 3851 & 0.000179984 & 3853 & 0.000179984 & 3863 & 0.000179984 & 3877 & 0.000179984 \\
\hline 3881 & 0.000179984 & 3889 & 0.000179984 & 3907 & 0.000179984 & 3911 & 0.000179984 \\
\hline 3917 & 0.000179984 & 3919 & 0.000179984 & 3923 & 0.000179984 & 3929 & 0.000179984 \\
\hline 3931 & 0.000179984 & 3943 & 0.000179984 & 3947 & 0.000179984 & 3967 & 0.000169986 \\
\hline 3989 & 0.000169986 & 4001 & 0.000169986 & 4003 & 0.000169986 & 4007 & 0.000169986 \\
\hline 4013 & 0.000169986 & 4019 & 0.000169986 & 4021 & 0.000169986 & 4027 & 0.000169986 \\
\hline 4049 & 0.000169986 & 4051 & 0.000169986 & 4057 & 0.000169986 & 4073 & 0.000169986 \\
\hline 4079 & 0.000169986 & 4091 & 0.000169986 & 4093 & 0.000169986 & 4099 & 0.000169986 \\
\hline 4111 & 0.000169986 & 4127 & 0.000169986 & 4129 & 0.000169986 & 4133 & 0.000169986 \\
\hline 4139 & 0.000169986 & 4153 & 0.000169986 & 4157 & 0.000169986 & 4159 & 0.000169986 \\
\hline 4177 & 0.000169986 & 4201 & 0.000169986 & 4211 & 0.000159987 & 4217 & 0.000159987 \\
\hline 4219 & 0.000159987 & 4229 & 0.000159987 & 4231 & 0.000159987 & 4241 & 0.000159987 \\
\hline 4243 & 0.000159987 & 4253 & 0.000159987 & 4259 & 0.000159987 & 4261 & 0.000159987 \\
\hline 4271 & 0.000159987 & 4273 & 0.000159987 & 4283 & 0.000159987 & 4289 & 0.000159987 \\
\hline 4297 & 0.000159987 & 4327 & 0.000159987 & 4337 & 0.000159987 & 4339 & 0.000159987 \\
\hline 4349 & 0.000159987 & 4357 & 0.000159987 & 4363 & 0.000159987 & 4373 & 0.000159987 \\
\hline 4391 & 0.000159987 & 4397 & 0.000159987 & 4409 & 0.000159987 & 4421 & 0.000159987 \\
\hline 4423 & 0.000159987 & 4441 & 0.000159987 & 4447 & 0.000159987 & 4451 & 0.000159987 \\
\hline 4457 & 0.000159987 & 4463 & 0.000159987 & 4481 & 0.000149989 & 4483 & 0.000149989 \\
\hline 4493 & 0.000149989 & 4507 & 0.000149989 & 4513 & 0.000149989 & 4517 & 0.000149989 \\
\hline 4519 & 0.000149989 & 4523 & 0.000149989 & 4547 & 0.000149989 & 4549 & 0.000149989 \\
\hline 4561 & 0.000149989 & 4567 & 0.000149989 & 4583 & 0.000149989 & 4591 & 0.000149989 \\
\hline 4597 & 0.000149989 & 4603 & 0.000149989 & 4621 & 0.000149989 & 4637 & 0.000149989 \\
\hline 4639 & 0.000149989 & 4643 & 0.000149989 & 4649 & 0.000149989 & 4651 & 0.000149989 \\
\hline 4657 & 0.000149989 & 4663 & 0.000149989 & 4673 & 0.000149989 & 4679 & 0.000149989 \\
\hline 4691 & 0.000149989 & 4703 & 0.000149989 & 4721 & 0.000149989 & 4723 & 0.000149989 \\
\hline 4729 & 0.000149989 & 4733 & 0.000149989 & 4751 & 0.000149989 & 4759 & 0.000149989 \\
\hline 4783 & 0.000139990 & 4787 & 0.000139990 & 4789 & 0.000139990 & 4793 & 0.000139990 \\
\hline 4799 & 0.000139990 & 4801 & 0.000139990 & 4813 & 0.000139990 & 4817 & 0.000139990 \\
\hline 4831 & 0.000139990 & 4861 & 0.000139990 & 4871 & 0.000139990 & 4877 & 0.000139990 \\
\hline 4889 & 0.000139990 & 4903 & 0.000139990 & 4909 & 0.000139990 & 4919 & 0.000139990 \\
\hline 4931 & 0.000139990 & 4933 & 0.000139990 & 4937 & 0.000139990 & 4943 & 0.000139990 \\
\hline
\end{tabular}


A computer verification for the value of the Topological Entropy... 469

Entropy Values for Prime Numbers Between 4951 and 6079

\begin{tabular}{|c|c|c|c|c|c|c|c|}
\hline Value & Entropy & Value & Entropy & Value & Entropy & Value & Entropy \\
\hline 4951 & 0.000139990 & 4957 & 0.000139990 & 4967 & 0.000139990 & 4969 & 0.000139990 \\
\hline 4973 & 0.000139990 & 4987 & 0.000139990 & 4993 & 0.000139990 & 4999 & 0.000139990 \\
\hline 5003 & 0.000139990 & 5009 & 0.000139990 & 5011 & 0.000139990 & 5021 & 0.000139990 \\
\hline 5023 & 0.000139990 & 5039 & 0.000139990 & 5051 & 0.000139990 & 5059 & 0.000139990 \\
\hline 5077 & 0.000139990 & 5081 & 0.000139990 & 5087 & 0.000139990 & 5099 & 0.000139990 \\
\hline 5101 & 0.000139990 & 5107 & 0.000139990 & 5113 & 0.000139990 & 5119 & 0.000139990 \\
\hline 5147 & 0.000129992 & 5153 & 0.000129992 & 5167 & 0.000129992 & 5171 & 0.000129992 \\
\hline 5179 & 0.000129992 & 5189 & 0.000129992 & 5197 & 0.000129992 & 5209 & 0.000129992 \\
\hline 5227 & 0.000129992 & 5231 & 0.000129992 & 5233 & 0.000129992 & 5237 & 0.000129992 \\
\hline 5261 & 0.000129992 & 5273 & 0.000129992 & 5279 & 0.000129992 & 5281 & 0.000129992 \\
\hline 5297 & 0.000129992 & 5303 & 0.000129992 & 5309 & 0.000129992 & 5323 & 0.000129992 \\
\hline 5333 & 0.000129992 & 5347 & 0.000129992 & 5351 & 0.000129992 & 5381 & 0.000129992 \\
\hline 5387 & 0.000129992 & 5393 & 0.000129992 & 5399 & 0.000129992 & 5407 & 0.000129992 \\
\hline 5413 & 0.000129992 & 5417 & 0.000129992 & 5419 & 0.000129992 & 5431 & 0.000129992 \\
\hline 5437 & 0.000129992 & 5441 & 0.000129992 & 5443 & 0.000129992 & 5449 & 0.000129992 \\
\hline 5471 & 0.000129992 & 5477 & 0.000129992 & 5479 & 0.000129992 & 5483 & 0.000129992 \\
\hline 5501 & 0.000129992 & 5503 & 0.000129992 & 5507 & 0.000129992 & 5519 & 129992 \\
\hline 5521 & 0.000129992 & 5527 & 0.000129992 & 5531 & 0.000129992 & 5557 & 0.000119993 \\
\hline 5563 & 0.000119993 & 5569 & 0.000119993 & 5573 & 0.000119993 & 5581 & 0.000119993 \\
\hline 5591 & 0.000119993 & 5623 & 0.000119993 & 5639 & 0.000119993 & 5641 & 0.000119993 \\
\hline 5647 & 0.000119993 & 5651 & 0.000119993 & 5653 & 0.000119993 & 5657 & 0.000119993 \\
\hline 5659 & 0.000119993 & 5669 & 0.000119993 & 5683 & 0.000119993 & 5689 & 0.000119993 \\
\hline 5693 & 0.000119993 & 5701 & 0.000119993 & 5711 & 0.000119993 & 5717 & 0.000119993 \\
\hline 5737 & 0.000119993 & 5741 & 0.000119993 & 5743 & 0.000119993 & 5749 & 0.000119993 \\
\hline 5779 & 0.000119993 & 5783 & 0.000119993 & 5791 & 0.000119993 & 5801 & 0.000119993 \\
\hline 5807 & 0.000119993 & 5813 & 0.000119993 & 5821 & 0.000119993 & 5827 & 0.000119993 \\
\hline 5839 & 0.000119993 & 5843 & 0.000119993 & 5849 & 0.000119993 & 5851 & 0.000119993 \\
\hline 5857 & 0.000119993 & 5861 & 0.000119993 & 5867 & 0.000119993 & 5869 & 0.000119993 \\
\hline 5879 & 0.000119993 & 5881 & 0.000119993 & 5897 & 0.000119993 & 5903 & 0.000119993 \\
\hline 5923 & 0.000119993 & 5927 & 0.000119993 & 5939 & 0.000119993 & 5953 & 0.000119993 \\
\hline 5981 & 0.000119993 & 5987 & 0.000119993 & 6007 & 0.000119993 & 6011 & 0.000119993 \\
\hline 6029 & 0.000109994 & 6037 & 0.000109994 & 6043 & 0.000109994 & 6047 & 0.000109994 \\
\hline 6053 & 0.000109994 & 6067 & 0.000109994 & 6073 & 0.000109994 & 6079 & 0.000109994 \\
\hline
\end{tabular}


Entropy Values for Prime Numbers Between 6089 and 7237

\begin{tabular}{|c|c|c|c|c|c|c|c|}
\hline Value & Entropy & Value & Entropy & Value & Entropy & Value & Entropy \\
\hline 6089 & 0.000109994 & 6091 & 0.000109994 & 6101 & 0.000109994 & 6113 & 0.000109994 \\
\hline 6121 & 0.000109994 & 6131 & 0.000109994 & 6133 & 0.000109994 & 6143 & 0.000109994 \\
\hline 6151 & 0.000109994 & 6163 & 0.000109994 & 6173 & 0.000109994 & 6197 & 0.000109994 \\
\hline 6199 & 0.000109994 & 6203 & 0.000109994 & 6211 & 0.000109994 & 6217 & 0.000109994 \\
\hline 6221 & 0.000109994 & 6229 & 0.000109994 & 6247 & 0.000109994 & 6257 & 0.000109994 \\
\hline 6263 & 0.000109994 & 6269 & 0.000109994 & 6271 & 0.000109994 & 6277 & 0.000109994 \\
\hline 6287 & 0.000109994 & 6299 & 0.000109994 & 6301 & 0.000109994 & 6311 & 0.000109994 \\
\hline 6317 & 0.000109994 & 6323 & 0.000109994 & 6329 & 0.000109994 & 6337 & 0.000109994 \\
\hline 6343 & 0.000109994 & 6353 & 0.000109994 & 6359 & 0.000109994 & 6361 & 0.000109994 \\
\hline 6367 & 0.000109994 & 6373 & 0.000109994 & 6379 & 0.000109994 & 6389 & 0.000109994 \\
\hline 6397 & 0.000109994 & 6421 & 0.000109994 & 6427 & 0.000109994 & 6449 & 0.000109994 \\
\hline 6451 & 0.000109994 & 6469 & 0.000109994 & 6473 & 0.000109994 & 6481 & 0.000109994 \\
\hline 6491 & 0.000109994 & 6521 & 0.000109994 & 6529 & 0.000109994 & 6547 & 0.000109994 \\
\hline 6551 & 0.000109994 & 6553 & 0.000109994 & 6563 & 0.000109994 & 6569 & 0.000109994 \\
\hline 6571 & 0.000109994 & 6577 & 0.000109994 & 6581 & 0.000109994 & 6599 & 0.000109994 \\
\hline 6607 & $1.00 \mathrm{E}-004$ & 6619 & $1.00 \mathrm{E}-004$ & 6637 & $1.00 \mathrm{E}-004$ & 6653 & $1.00 \mathrm{E}-004$ \\
\hline 6659 & $1.00 \mathrm{E}-004$ & 6661 & $1.00 \mathrm{E}-004$ & 6673 & $1.00 \mathrm{E}-004$ & 6679 & $1.00 \mathrm{E}-004$ \\
\hline 6689 & $1.00 \mathrm{E}-004$ & 6691 & $1.00 \mathrm{E}-004$ & 6701 & $1.00 \mathrm{E}-004$ & 6703 & $1.00 \mathrm{E}-004$ \\
\hline 6709 & $1.00 \mathrm{E}-004$ & 6719 & $1.00 \mathrm{E}-004$ & 6733 & $1.00 \mathrm{E}-004$ & 6737 & $1.00 \mathrm{E}-004$ \\
\hline 6761 & $1.00 \mathrm{E}-004$ & 6763 & $1.00 \mathrm{E}-004$ & 6779 & $1.00 \mathrm{E}-004$ & 6781 & $1.00 \mathrm{E}-004$ \\
\hline 6791 & $1.00 \mathrm{E}-004$ & 6793 & $1.00 \mathrm{E}-004$ & 6803 & $1.00 \mathrm{E}-004$ & 6823 & $1.00 \mathrm{E}-004$ \\
\hline 6827 & $1.00 \mathrm{E}-004$ & 6829 & $1.00 \mathrm{E}-004$ & 6833 & $1.00 \mathrm{E}-004$ & 6841 & $1.00 \mathrm{E}-004$ \\
\hline 6857 & $1.00 \mathrm{E}-004$ & 6863 & $1.00 \mathrm{E}-004$ & 6869 & $1.00 \mathrm{E}-004$ & 6871 & $1.00 \mathrm{E}-004$ \\
\hline 6883 & $1.00 \mathrm{E}-004$ & 6899 & $1.00 \mathrm{E}-004$ & 6907 & $1.00 \mathrm{E}-004$ & 6911 & $1.00 \mathrm{E}-004$ \\
\hline 6917 & $1.00 \mathrm{E}-004$ & 6947 & $1.00 \mathrm{E}-004$ & 6949 & $1.00 \mathrm{E}-004$ & 6959 & $1.00 \mathrm{E}-004$ \\
\hline 6961 & $1.00 \mathrm{E}-004$ & 6967 & $1.00 \mathrm{E}-004$ & 6971 & $1.00 \mathrm{E}-004$ & 6977 & $1.00 \mathrm{E}-004$ \\
\hline 6983 & $1.00 \mathrm{E}-004$ & 6991 & $1.00 \mathrm{E}-004$ & 6997 & $1.00 \mathrm{E}-004$ & 7001 & $1.00 \mathrm{E}-004$ \\
\hline 7013 & $1.00 \mathrm{E}-004$ & 7019 & $1.00 \mathrm{E}-004$ & 7027 & $1.00 \mathrm{E}-004$ & 7039 & $1.00 \mathrm{E}-004$ \\
\hline 7043 & $1.00 \mathrm{E}-004$ & 7057 & $1.00 \mathrm{E}-004$ & 7069 & $1.00 \mathrm{E}-004$ & 7079 & $1.00 \mathrm{E}-004$ \\
\hline 7103 & $1.00 \mathrm{E}-004$ & 7109 & $1.00 \mathrm{E}-004$ & 7121 & $1.00 \mathrm{E}-004$ & 7127 & $1.00 \mathrm{E}-004$ \\
\hline 7129 & $1.00 \mathrm{E}-004$ & 7151 & $1.00 \mathrm{E}-004$ & 7159 & $1.00 \mathrm{E}-004$ & 7177 & $1.00 \mathrm{E}-004$ \\
\hline 7187 & $1.00 \mathrm{E}-004$ & 7193 & $1.00 \mathrm{E}-004$ & 7207 & $1.00 \mathrm{E}-004$ & 7211 & $1.00 \mathrm{E}-004$ \\
\hline 7213 & $1.00 \mathrm{E}-004$ & 7219 & $1.00 \mathrm{E}-004$ & 7229 & $1.00 \mathrm{E}-004$ & 7237 & $1.00 \mathrm{E}-004$ \\
\hline
\end{tabular}


A computer verification for the value of the Topological Entropy... 471

Entropy Values for Prime Numbers Between 7243 and 8447

\begin{tabular}{|c|c|c|c|c|c|c|c|}
\hline Value & Entropy & Value & Entropy & Value & Entropy & Value & Entropy \\
\hline 7243 & $00 \mathrm{E}-004$ & 7247 & $00 \mathrm{E}-004$ & 7253 & $1.00 \mathrm{~L}-004$ & 7283 & $1.00 \mathrm{E}-004$ \\
\hline 7297 & $9.00 \mathrm{E}-005$ & 7307 & $.00 \mathrm{E}-005$ & 7309 & $9.00 \mathrm{E}-005$ & 321 & $9.00 \mathrm{E}-005$ \\
\hline 7331 & $9.00 \mathrm{E}-005$ & 7333 & $9.00 \mathrm{E}-005$ & 7349 & $9.00 \mathrm{E}-005$ & 7351 & $9.00 \mathrm{E}-005$ \\
\hline 7369 & $9.00 \mathrm{E}-005$ & 7393 & $9.00 \mathrm{E}-005$ & 7411 & $9.00 \mathrm{E}-005$ & 7417 & $9.00 \mathrm{E}-005$ \\
\hline 7433 & $9.00 \mathrm{E}-005$ & 7451 & $9.00 \mathrm{E}-005$ & 7457 & $9.00 \mathrm{E}-005$ & 7459 & $9.00 \mathrm{E}-005$ \\
\hline 7477 & $9.00 \mathrm{E}-005$ & 7481 & $9.00 \mathrm{E}-005$ & 7487 & $9.00 \mathrm{E}-005$ & 7489 & $9.00 \mathrm{E}-005$ \\
\hline 7499 & $9.00 \mathrm{E}-005$ & 7507 & $9.00 \mathrm{~F}$ & 7517 & 05 & 7523 & -005 \\
\hline 7529 & $9.00 \mathrm{E}-005$ & 7537 & $9.00 \mathrm{E}-005$ & 7541 & $9.00 \mathrm{E}-005$ & 7547 & $9.00 \mathrm{E}-005$ \\
\hline 7549 & $9.00 \mathrm{E}-005$ & 7559 & $9.00 \mathrm{E}-005$ & 7561 & 9.001 & 7573 & $9.00 \mathrm{E}-005$ \\
\hline 7577 & $9.00 \mathrm{E}-005$ & 7583 & $9.00 \mathrm{E}-005$ & 7589 & $9.00 \mathrm{~F}$ & 7591 & -005 \\
\hline 7603 & $9.00 \mathrm{E}-005$ & 7607 & $9.00 \mathrm{E}-005$ & 7621 & $9.00 \mathrm{E}-005$ & 7639 & $9.00 \mathrm{E}-005$ \\
\hline 7643 & 9.00 & 7649 & 9.00 & 7669 & 05 & 7673 & 005 \\
\hline 7681 & $9.00 \mathrm{E}-005$ & 7687 & $9.00 \mathrm{E}-005$ & 7691 & 9.00 & 7699 & $9.00 \mathrm{E}-005$ \\
\hline 7703 & $9.00 \mathrm{E}-005$ & 7717 & -005 & 7723 & 9.00 & 7727 & -005 \\
\hline 7741 & $9.00 \mathrm{E}-005$ & 7753 & $9.00 \mathrm{E}-005$ & 7757 & 9.00 & 7759 & $9.00 \mathrm{E}-005$ \\
\hline 7789 & $9.00 \mathrm{E}-005$ & 7793 & $9.00 \mathrm{E}-005$ & 7817 & $9.00 \mathrm{E}-005$ & 7823 & $9.00 \mathrm{E}-005$ \\
\hline 7829 & $9.00 \mathrm{E}-005$ & 7841 & 9.00 & 7853 & 9.00 & 7867 & -005 \\
\hline 7873 & $9.00 \mathrm{E}-005$ & 7877 & $9.00 \mathrm{E}-005$ & 7879 & $9.00 \mathrm{E}-005$ & 7883 & $9.00 \mathrm{E}-005$ \\
\hline 7901 & $9.00 \mathrm{E}-005$ & 7907 & 9.00 & 7919 & $9.00 \mathrm{E}-005$ & 7927 & $9.00 \mathrm{E}-005$ \\
\hline 7933 & $9.00 \mathrm{E}-005$ & 7937 & 9.00 & 7949 & $9.00 \mathrm{E}-005$ & 7951 & $9.00 \mathrm{E}-005$ \\
\hline 7963 & $9.00 \mathrm{E}-005$ & 7993 & $9.00 \mathrm{E}-005$ & 8009 & $9.00 \mathrm{E}-005$ & 8011 & $9.00 \mathrm{E}-005$ \\
\hline 8017 & $9.00 \mathrm{E}-005$ & 8039 & $9.00 \mathrm{E}-005$ & 8053 & $9.00 \mathrm{E}-005$ & 8059 & $9.00 \mathrm{E}-005$ \\
\hline 8069 & $9.00 \mathrm{E}-005$ & 8081 & $9.00 \mathrm{E}-005$ & 8087 & $9.00 \mathrm{E}-005$ & 8089 & $9.00 \mathrm{E}-005$ \\
\hline 8093 & $9.00 \mathrm{E}-005$ & 8101 & $9.00 \mathrm{E}-005$ & 8111 & $9.00 \mathrm{E}-005$ & 8117 & $9.00 \mathrm{E}-005$ \\
\hline 8123 & $9.00 \mathrm{E}-005$ & 8147 & 9.00 & 8161 & $8.00 \mathrm{E}-005$ & 8167 & E-005 \\
\hline 8171 & $8.00 \mathrm{E}-005$ & 8179 & $8.00 \mathrm{E}-005$ & 8191 & $8.00 \mathrm{E}-005$ & 8209 & 8.00E-005 \\
\hline 8219 & $8.00 \mathrm{E}-005$ & 8221 & $8.00 \mathrm{E}-005$ & 8231 & $8.00 \mathrm{E}-005$ & 8233 & $8.00 \mathrm{E}-005$ \\
\hline 8237 & $8.00 \mathrm{E}-005$ & 8243 & $8.00 \mathrm{E}-005$ & 8263 & $8.00 \mathrm{E}-005$ & 8269 & $8.00 \mathrm{E}-005$ \\
\hline 8273 & $8.00 \mathrm{E}-005$ & 8287 & $8.00 \mathrm{E}-005$ & 8291 & $8.00 \mathrm{E}-005$ & 8293 & $8.00 \mathrm{E}-005$ \\
\hline 8297 & $8.00 \mathrm{E}-005$ & 8311 & $8.00 \mathrm{E}-005$ & 8317 & $8.00 \mathrm{E}-005$ & 8329 & $8.00 \mathrm{E}-005$ \\
\hline 8353 & $8.00 \mathrm{E}-005$ & 8363 & $8.00 \mathrm{E}-005$ & 8369 & $8.00 \mathrm{E}-005$ & 8377 & $8.00 \mathrm{E}-005$ \\
\hline 8387 & $8.00 \mathrm{E}-005$ & 8389 & 8.00 & 8419 & 8.00 & 8423 & $8.00 \mathrm{E}-005$ \\
\hline 8429 & $8.00 \mathrm{E}-005$ & 8431 & $8.00 \mathrm{E}-005$ & 8443 & $8.00 \mathrm{E}-005$ & 8447 & $8.00 \mathrm{E}-005$ \\
\hline
\end{tabular}


Entropy Values for Prime Numbers Between 8461 and 9697

\begin{tabular}{|c|c|c|c|c|c|c|c|}
\hline Value & Entropy & Value & Entropy & Value & Entropy & Value & Entropy \\
\hline 8461 & 001 & 0 & 00 & 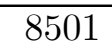 & Ju & 10 & 0 \\
\hline 8521 & 5 & 27 & 5 & 37 & 5 & 39 & 05 \\
\hline 8543 & 8.00E-005 & 8563 & 8.00E-005 & 8573 & $8.00 \mathrm{E}-005$ & 8581 & 8.00E-005 \\
\hline 8597 & $8.00 \mathrm{E}-005$ & 8599 & $8.00 \mathrm{E}-005$ & 8609 & $8.00 \mathrm{E}-005$ & 8623 & 8.00 \\
\hline 8627 & 8.00E-005 & 8629 & $8.00 \mathrm{E}-005$ & 8641 & $8.00 \mathrm{E}-005$ & 8647 & -005 \\
\hline 8663 & $8.00 \mathrm{E}-005$ & 8669 & $8.00 \mathrm{~F}$ & 8677 & $8.00 \mathrm{E}-005$ & 8681 & -005 \\
\hline 8689 & $8.00 \mathrm{E}-005$ & 993 & $8.00 \mathrm{~F}$ & 8699 & $.00 \mathrm{E}-005$ & 707 & -005 \\
\hline 8713 & $8.00 \mathrm{E}-005$ & 8719 & 8.00 & 8731 & $8.00 \mathrm{E}-005$ & 8737 & -005 \\
\hline 8741 & $8.00 \mathrm{E}-005$ & 8747 & 8.001 & 8753 & $8.00 \mathrm{E}-005$ & 8761 & -005 \\
\hline 8779 & $8.00 \mathrm{E}-005$ & 33 & 8.00 & 8803 & 8.00 & 807 & 005 \\
\hline 8819 & $8.00 \mathrm{E}-005$ & 8821 & 8.001 & 8831 & $8.00 \mathrm{E}-005$ & 8837 & $8.00 \mathrm{E}-005$ \\
\hline 8839 & 8.00 & & & 361 & & 63 & 05 \\
\hline 8867 & 8.001 & 87 & 8.00 & 8893 & -005 & 8923 & -005 \\
\hline 8929 & $8.00 \mathrm{E}-005$ & 8933 & 8.00 & 8941 & 8.00 & 8951 & -005 \\
\hline 8963 & 8.00 & 99 & 8.00 & 8971 & 05 & 999 & 005 \\
\hline 9001 & $8.00 \mathrm{E}-005$ & 9007 & 8.00 & 9011 & $8.00 \mathrm{E}-005$ & 9013 & -005 \\
\hline 9029 & 8.00 & 11 & 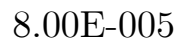 & 9043 & 8.0 & 9049 & 05 \\
\hline 9059 & 8.00 & 9067 & 05 & 9091 & 8.0 & 9103 & -005 \\
\hline 9109 & 8.00 & 9127 & 8.0 & 9133 & 8.00 & 9137 & -005 \\
\hline 9151 & 8.00E-005 & 9157 & 8.00 & 9161 & -005 & 173 & 005 \\
\hline 9181 & 8.00 & 187 & 8.0 & 9199 & 8.0 & 9203 & -005 \\
\hline 9209 & 8.00 & 9221 & 8.00 & 9227 & 8.00 & 9239 & -005 \\
\hline 9241 & 8.00 & 9257 & 7.0 & 9277 & 7.00E-005 & 9281 & -005 \\
\hline 9283 & 7.00E-005 & 9293 & 7.00 & 9311 & 7.00E-005 & 9319 & -005 \\
\hline 9323 & 7.00 & 9337 & 7.0 & 9341 & 7.00 & 9343 & -005 \\
\hline 9349 & 7.00 & 9371 & 7.0 & 9377 & 7.00 & 391 & -005 \\
\hline 9397 & 7.00 & 9403 & 05 & 9413 & 7.00E-005 & 9419 & -005 \\
\hline 9421 & 7.00E-005 & 9431 & 7.00E-005 & 9433 & 7.00E-005 & 9437 & 7.00E-005 \\
\hline 9439 & $7.00 \mathrm{E}-005$ & 9461 & $7.00 \mathrm{H}$ & 9463 & 7.00E-005 & 9467 & $7.00 \mathrm{E}-005$ \\
\hline 9473 & $7.00 \mathrm{E}-005$ & 9479 & 7.00 & 9491 & 7.00E-005 & 9497 & $7.00 \mathrm{E}-005$ \\
\hline 9511 & $7.00 \mathrm{E}-005$ & 9521 & $7.00 \mathrm{E}-005$ & 9533 & 7.00E-005 & 9539 & 7.00E-005 \\
\hline 9547 & 7.00E-005 & 9551 & 7.00 & 9587 & 7.00E-005 & 9601 & 7.00E-005 \\
\hline 9613 & $7.00 \mathrm{E}-005$ & 9619 & $7.00 \mathrm{E}-005$ & 9623 & 7.00E-005 & 9629 & $7.00 \mathrm{E}-005$ \\
\hline 9631 & $7.00 \mathrm{E}-005$ & 9643 & $7.00 \mathrm{E}-005$ & 9649 & $7.00 \mathrm{E}-005$ & 9661 & $7.00 \mathrm{E}-005$ \\
\hline 9677 & 7.00E-005 & 9679 & $7.00 \mathrm{E}-005$ & 9689 & 7.00E-005 & 9697 & $7.00 \mathrm{E}-005$ \\
\hline
\end{tabular}


A computer verification for the value of the Topological Entropy... 473

Entropy Values for Prime Numbers Between 9719 and 9973

\begin{tabular}{|c|l|l|l|l|l|l|l|}
\hline Value & Entropy & Value & Entropy & Value & Entropy & Value & Entropy \\
\hline 9719 & $7.00 \mathrm{E}-005$ & 9721 & $7.00 \mathrm{E}-005$ & 9733 & $7.00 \mathrm{E}-005$ & 9739 & $7.00 \mathrm{E}-005$ \\
9743 & $7.00 \mathrm{E}-005$ & 9749 & $7.00 \mathrm{E}-005$ & 9767 & $7.00 \mathrm{E}-005$ & 9769 & $7.00 \mathrm{E}-005$ \\
9781 & $7.00 \mathrm{E}-005$ & 9787 & $7.00 \mathrm{E}-005$ & 9791 & $7.00 \mathrm{E}-005$ & 9803 & $7.00 \mathrm{E}-005$ \\
9811 & $7.00 \mathrm{E}-005$ & 9817 & $7.00 \mathrm{E}-005$ & 9829 & $7.00 \mathrm{E}-005$ & 9833 & $7.00 \mathrm{E}-005$ \\
9839 & $7.00 \mathrm{E}-005$ & 9851 & $7.00 \mathrm{E}-005$ & 9857 & $7.00 \mathrm{E}-005$ & 9859 & $7.00 \mathrm{E}-005$ \\
9871 & $7.00 \mathrm{E}-005$ & 9883 & $7.00 \mathrm{E}-005$ & 9887 & $7.00 \mathrm{E}-005$ & 9901 & $7.00 \mathrm{E}-005$ \\
9907 & $7.00 \mathrm{E}-005$ & 9923 & $7.00 \mathrm{E}-005$ & 9929 & $7.00 \mathrm{E}-005$ & 9931 & $7.00 \mathrm{E}-005$ \\
9941 & $7.00 \mathrm{E}-005$ & 9949 & $7.00 \mathrm{E}-005$ & 9967 & $7.00 \mathrm{E}-005$ & 9973 & $7.00 \mathrm{E}-005$ \\
\hline
\end{tabular}




\section{References}

[1] Adler, R. L.; Konheim, A.G.; Mc Andrew, M.H. Topological Entropy Transactiones of the A.M.S. vol 144, pp. 309-319, (1965).

[2] Alekseev, V. M. Quasirandom Dynamical Systems Math. USSR Sbornik - New Series Vol. $5 N^{o}$ 1, (1968).

[3] Alseda, Lluís; Llibre, Jaume; Misiurewicz, Michal Combinatorial dynamics and entropy in dimension one. Second edition. Advenced Series in Nonlinear Dynamics, 5. World Scientific Publishing Co., Inc., River Edge, NJ, xvi+15, pp. ISBN: 981-02-4053-8, (2000).

[4] Aranzubia, S.Hacia una demostración de la arco conexidad de la isentropa de entropía cero de la familia cuadrática de Lorenz lexicográfica: burbujas de entropía constante y cotas superiores para burbujas las de entropía cero Tesis de doctorado Universidad de Santiago de Chile, (2015)

[5] Aranzubia, S.; Labarca R. A Formula for the Boundary of Chaos in the Lexicographical Scenario and Applications to the Bifurcation Diagram of the Standard Two Parameter Family of Quadratic Increasing-Increasing Lorenz maps. Discrete and Continuous Dynamical Systems-Series A., Vol. 38, $N^{o}$ 4, pp. 1745-1776, (2018).

[6] Block,L; Guckenheimer, J.; Misiurewicz, M; Young, L.S. Periodic points and topological entropy. Lectures Notes in Mathematics. $N^{o} 819$, Springer Verlag, (1978).

[7] Bamón,R.; Labarca, R.; Mañé R.; Pacifico, M.J.The explosion of singular cycles Publ. Math. IHES Vol 78, pp 207-232, (1993).

[8] Bruin, H.; Van Strien, S. Monotonicity of Entropy for Real Multimodal Maps Journal of the American Mathematical Society, Vol 28, No 1, pp. $1-61,(2015)$.

[9] de Melo W., Van Strien S. Lectures on One Dimensional Dynamics Springer Verlag, (1993).

[10] Francis, J. G. F.; The QR Transformation, I. The Computer Journal, 4(3), pp. 265-271, (1961). 
A computer verification for the value of the Topological Entropy... 475

[11] The GNU Scientific Library $\quad$ (GSL); http://www.gnu.org/software/gsl/

[12] Guckenheimer J., Williams R. F. Structural Stability of Lorenz Attractors Publ. Math. IHES 50, pp 59-72, (1979).

[13] Labarca, R. Bifurcation of Contracting Singular Cycles Ann. Scient. Ec. Norm. Sup. $4^{e}$ serie, t. 28, pp 705-745, (1995).

[14] Labarca, R. A note on the topological classification of Lorenz maps on the interval. Topics in symbolic dynamics an applications, London Math. Soc. Lect. Not. Ser. 279, Cambridge Univ. Press. pp. 229-245, (2000).

[15] Labarca, R. Unfolding singular cycles. Notas Soc. Mat. Chile (N5) No 1, pp. 38-71, (2001).

[16] Labarca, R,La Entropía topológica, propiedades generales y algunos cálculos en el caso del shift de Milnor-Thurston.Con la colaboración de Solange Aranzubia V. y Erick Inda R. Libro de 150 páginas publicado en la serie de las Escuelas Venezolanas de Matemáticas, con comité editorial, de Ediciones IVIC. Instituto Venezolano de Investigaciones Científicas, (2011)

[17] Labarca,R.; Moreira C. Bifurcation of the Essential Dynamics of Lorenz Maps of the real Line and the Bifurcation Scenario for the Linear family Scientia Ser A Math. Sci (N-S), Vol. 7, pp. 13-29, (2001).

[18] Labarca, R.; Moreira C. Bifurcation of the Essential Dynamics of Lorenz Maps and applications to Lorenz Like Flows: Contributions to the study of the Expanding Case. Bol. Soc. Bros. Mat. (N-S), Vol. 32, pp. 107-144, (2001).

[19] Labarca, R.; Moreira C. Essential Dynamics for Lorenz Maps on the real line and Lexicographical World. Ann. de L'Institut H. Poincaré Anal. non Linneaire, Vol. 23, pp. 683-694, (2006).

[20] Labarca, R.; Moreira, C.Bifurcation of the essential dynamics of Lorenz Maps on the real line and the bifurcation scenario for Lorenz like flows: The Contracting Case Proyecciones. Journal of Mathematics, Vol 29, $N^{o} .3$, pp. 241-289 (2010). 
[21] Labarca R., Plaza S. Bifurcations of discontinuous maps of the interval and palindromic numbers. Boletín de la Sociedad Matemática Mexicana(3), Vol 5., pp. 87-104, (2001)

[22] Labarca, R.; San Martín, B. Prevalence of hyperbolicity for complex singular cycles. Bol. Soc. Brasil. Mat. (N5) 28, $N^{o} 2$ pp. 343-362, (1997).

[23] Labarca, R.; Vásquez, L. On the Characterization of the kneading sequences associated to Lorenz maps of the interval and to orientation preserving homeomorphism of the circle Boletín de la Sociedad Matemática Méxicana (3), Vol. $16 N^{o} .2$, pp. 103-118, (2010).

[24] Labarca R., Vásquez L. On the Characterization of the Kneading Sequences Associated to Lorenz Maps of the Interval. Bol. Soc. Bras. Mat. (NS), Vol. $43 N^{o}$ 2, pp. 221-245, (2012).

[25] Moreira, C. Maximal invariant sets for restriction of tent and unimodal maps. Qual. Thory Dyn. Syst, 2, No2, pp. 385-398, (2001).

[26] Metropolis, N.; Stein, M. L. ; Stein, P.R. "Stabe States of a nonlinear transformation.". Numerisch Mathematik 10, pp. 1-19, (1967).

[27] Metropolis, N.; Stein, M. L.; Stein, P. R. "On finite limit sets for transformations on the unit interval.". Journal of combinatorial Theory (A) 15, pp. 25-44, (1973).

[28] Milnor, J. Remarks on iterated cubic maps Experiment. Math. 1, $N^{o}$ 1, pp. 5-24 MR1181083, (1992).

[29] Milnor, J. ; Thurston, W. On iterated maps on the interval. Lect. Notes in Math 1342 pp 465-563 Springer Verlag, (1988).

[30] Misiurewicz, M. On non continuity of topological entropy Bull. Acad. Polon. Sci. Ser. Sci. Math. Astronom. Phys. 19, pp. 319-320, (1971).

[31] Sait Pierre, Matthias Topological and measurable dynamics of Lorenz Maps. Dissertationes Mathematicae (ROZPRAWY MATEMATYCZNE) Polska Akademie Nauk, Institut Matematiczny. CCCLXXXII, (1999).

[32] Silnikov, L.P.A Contribution to the problem of the structure of an extended neighborhood of a rough equilibrium state of saddle-focus type, Math. USSR Sbornik, Vol. $10 N^{o} 1,(1970)$. 
A computer verification for the value of the Topological Entropy... 477

[33] Smale, S. Differentiable dynamical systems. Bull. Amer. Math. Soc, 73, pp. 747-819, (1967).

[34] Zacks, Mikhail A. Scaling Properties and renormalization invariants for the "homoclinic quasiperiodicity". Physyca D, Vol 92, pp. 300-316, (1993).

\section{S. Aranzubia}

Escuela de Matemática y Estadística

Universidad Central de Chile

Santiago

Chile

e-mail : solange.aranzubia@gmail.com

\section{R. Carvajal}

Departamento de Matematica y Ciencia de la Computacin Universidad de Santiago de Chile

Casilla 307 Correo 2

Santiago

Chile

e-mail : ruben.carvajal@usach.cl

and

\section{R. Labarca}

Departamento de Matematica y Ciencia de la Computacin Universidad de Santiago de Chile

Casilla 307 Correo 2

Santiago

Chile

e-mail : rafael.labarca@usach.cl 